\title{
Neutrino-nucleon cross-section model tuning in GENIE v3
}

\author{
Júlia Tena-Vidal $\odot,{ }^{1}$ Costas Andreopoulos, ${ }^{1,2}$ Adi Ashkenazi,${ }^{3}$ Christopher Barry, ${ }^{1}$ Steve Dennis, ${ }^{1, *}$ Steve Dytman, ${ }^{4}$ \\ Hugh Gallagher, ${ }^{5}$ Steven Gardiner, ${ }^{6}$ Walter Giele, ${ }^{6}$ Robert Hatcher, ${ }^{6}$ Or Hen, ${ }^{3}$ Libo Jiang, ${ }^{4, \dagger}$ Igor D. Kakorin, ${ }^{7}$ \\ Konstantin S. Kuzmin, ${ }^{7}$ Anselmo Meregaglia, ${ }^{8}$ Vadim A. Naumov, ${ }^{7}$ Afroditi Papadopoulou, ${ }^{3}$ Gabriel Perdue, ${ }^{6}$ \\ Marco Roda $\odot,{ }^{1}$ Vladyslav Syrotenko, ${ }^{5}$ and Jeremy Wolcott ${ }^{5}$
}

(GENIE Collaboration)

\author{
${ }^{1}$ University of Liverpool, Department of Physics, Liverpool L69 7ZE, United Kingdom \\ ${ }^{2}$ Science and Technology Facilities Council, Rutherford Appleton Laboratory, \\ Particle Physics Department, Oxfordshire OX11 OQX, United Kingdom \\ ${ }^{3}$ Massachusetts Institute of Technology, Department of Physics, Cambridge, Massachusetts 02139, USA \\ ${ }^{4}$ University of Pittsburgh, Department of Physics and Astronomy, Pittsburgh, Pennsylvania 15260, USA \\ ${ }^{5}$ Tufts University, Department of Physics and Astronomy, Medford, Massachusetts 02155, USA \\ ${ }^{6}$ Fermi National Accelerator Laboratory, Batavia, Illinois 60510, USA \\ ${ }^{7}$ Joint Institute for Nuclear Research (JINR), Dubna, Moscow region 141980, Russia \\ ${ }^{8}$ CENBG, Université de Bordeaux, CNRS/IN2P3, 33175 Gradignan, France
}

(Received 14 June 2021; accepted 1 September 2021; published 20 October 2021)

\begin{abstract}
We summarize the results of a study performed within the GENIE global analysis framework, revisiting the GENIE bare-nucleon cross-section tuning and, in particular, the tuning of (a) the inclusive cross section, (b) the cross section of low-multiplicity inelastic channels (single-pion and double-pion production), and (c) the relative contributions of resonance and nonresonance processes to these final states. The same analysis was performed with several different comprehensive cross-section model sets available in GENIE Generator v3. In this work we perform a careful investigation of the observed tensions between exclusive and inclusive data, and install analysis improvements to handle systematics in historic data. All tuned model configurations discussed in this paper are available through public releases of the GENIE Generator. With this paper we aim to support the consumers of these physics tunes by providing comprehensive summaries of our alternate model constructions, of the relevant datasets and their systematics, and of our tuning procedure and results.
\end{abstract}

DOI: 10.1103/PhysRevD.104.072009

\section{INTRODUCTION}

GENIE is an international collaboration of scientists working on a global analysis of neutrino scattering data and on the incorporation of modern theoretical inputs and experimental data into robust and predictive semiempirical comprehensive neutrino interaction simulations. GENIE develops and maintains a suite of well-known software products for the experimental neutrino community, which includes its popular Generator product [1]. With the recent release of the GENIE Generator v3, a substantial change in

\footnotetext{
Now at the University of Cambridge.

${ }^{\dagger}$ Virginia Polytechnic Institute and State University.
}

Published by the American Physical Society under the terms of the Creative Commons Attribution 4.0 International license. Further distribution of this work must maintain attribution to the author(s) and the published article's title, journal citation, and DOI. Funded by SCOAP ${ }^{3}$. the way that the GENIE Collaboration approaches the process of developing, validating, characterizing, tuning, and releasing comprehensive neutrino interaction simulations came into sharp focus. The focus of the GENIE Collaboration has always been the development of universal comprehensive models, handling all probes and targets and simulating all processes across the entire kinematic phase space relevant for neutrino experiments. Previously, the GENIE Collaboration released a single, preferred (default) comprehensive model that reflected our current understanding of the most predictive, robust, and selfconsistent model that could be built out of GENIE neutrino interaction modeling elements. Whereas many other alternative modeling elements were made available to users, they had to be enabled by individual users through an errorprone procedure that could bring substantial physics and logical inconsistencies, invalidate procedures for addressing double counting issues, and damage the level of agreement with data, often in ways that were unsuspected 
by users that had a narrow focus on some particular modeling aspect, and lacked the GENIE tools and procedures to fully characterize a comprehensive model. To address this, and in response to the community demand for alternative models, GENIE has released a number of comprehensive model configurations (CMCs) and is in the process of constructing several more. All such configurations, that are easily invoked and run out of the box, combine modeling elements in a way that is as consistent as possible, and are validated, characterized, and tuned as a whole. This important development was underpinned by a substantial upgrade of GENIE capabilities for systematic model validation, model characterization through comparisons to large collections of complementary scattering data with neutrino, charged lepton and hadron probes, and the development of an advanced global analysis of scattering data.

The GENIE global analysis was made possible through the continued development of curated data archives, and the successful large-scale refactoring and interfacing to the Professor tool [2] of a very extensive set of GENIE codes, that implement comparisons to data within a framework that allows the efficient manipulation of large ensembles of simulated events produced from a constellation of alternative models. The interface to the Professor tool enabled the efficient implementation of complex multiparameter brute-force scans and removed substantial global analysis limitations by decoupling it from event reweighting procedures that, for all but the most trivial aspects of our physics domain, require substantial development time and are not exact, or even possible at all. Professor 'reduces the exponentially expensive process of brute-force tuning to a scaling closer to a power law in the number of parameters, while allowing for massive parallelization' [3]. The Professor package has been extensively used for the tuning of Monte Carlo generators in the collider community.

The above developments allowed the GENIE Collaboration to fulfil its dual purpose described in its mission statement; GENIE develops a popular Monte Carlo event generation platform and implements, within its platform, universal and comprehensive physics simulations for lepton scattering, as well as simulations for several Beyond the Standard Model processes. But, in addition, and separately from the previous mission, GENIE develops a global analysis of scattering data for the tuning and uncertainty characterization of comprehensive neutrino interaction models. The GENIE Generator is the main outlet for the GENIE global analysis results, and our goal is that, for each supported comprehensive model, several selected tuned versions shall be made available.

Typically, nuclear modifications to the cross section are computed separately, and the decomposition of the total cross section into the possible exclusive final states proceeds via separate hadronization, intranuclear rescattering, and particle decay codes. Therefore, bare-nucleon cross sections are a crucial first modeling component to tune in the process of building a global fit of all relevant scattering data. Tunes for several aspects of GENIE modeling, including neutrino-induced hadronization and nuclear cross sections for low-multiplicity channels, are near completion and will be released and published in the future. This paper summarizes the results of the first analysis performed within the GENIE global analysis framework, revisiting the GENIE bare-nucleon cross-section tune and, in particular, the tuning of the empirical nonresonance background contribution to one- and twopion final states. A similar, albeit much simpler, analysis underpinned the tune of the well-known and widely-used comprehensive model that was included as the default model throughout the very long GENIE v2 series of releases. At that time, not sufficiently explored and understood tensions between inclusive and exclusive data, and an executive decision to anchor the GENIE v2 model on inclusive data, led to some expected and well-known discrepancies with exclusive data that were increasingly brought into focus as new experiments started performing increasingly precise measurements of low-multiplicity exclusive final states [4]. Here, we perform a careful investigation of the observed tensions between exclusive and inclusive data, retune the bare-nucleon cross-section model for all GENIE comprehensive models available in GENIE v3, and provide best-fit values and correlations for several parameters influencing the GENIE bare-nucleon cross sections. The work presented here was based on the model implementations of GENIE v3.0.6 (released on 23 July 2019), and the results of this work will be included in the GENIE v3.2.0 release. Preliminary versions of this work appeared in earlier releases of the GENIE v3 series (v3.0.0-v3.0.6).

In Sec. II, we summarize relevant aspects of the free nucleon cross-section modeling in GENIE, while in Sec. III we provide further details for the construction of comprehensive GENIE models considered in this work. In Sec. IV we provide details of the datasets, parametrization of the model, and data uncertainties for this particular tune. Section V describes the tuning procedure as well as the statistical methodology used. Finally, our tuning results are presented in Sec. VI.

\section{BARE NUCLEON CROSS-SECTION MODELING IN GENIE}

In very simplified terms, neglecting diffractive production, as well as $|\Delta S|=1$ and $|\Delta C|=1$ processes, the total inelastic differential cross section for neutrino scattering off bare nucleons, $d^{2} \sigma^{\text {inel }} / d Q^{2} d W$, is computed in GENIE as

$$
\frac{d^{2} \sigma^{\text {inel }}}{d Q^{2} d W}= \begin{cases}\frac{d^{2} \sigma^{\mathrm{RES}}}{d Q^{2} d W}+\frac{d^{2} \sigma^{\mathrm{SIS}}}{d Q^{2} d W} & \text { for } W<W_{\text {cut }} \\ \frac{d^{2} \sigma^{\mathrm{DIS}}}{d Q^{2} d W} & \text { for } W \geq W_{\text {cut }} .\end{cases}
$$


The term $d^{2} \sigma^{\mathrm{RES}} / d Q^{2} d W$ represents the contribution from all low-multiplicity inelastic channels proceeding via resonance production (RES) and, in present versions of GENIE, it is computed as an incoherent sum over several resonances. The resonances included in GENIE v3 are the ones specified by the Rein-Seghal paper [5]. The nine lightest $N^{*}$ and the eight lightest $\Delta$ labeled by the PDG with three or four stars are considered. The following resonances are included in GENIE v3: $N(1440), N(1520), N(1535)$, $N(1650), \quad N(1675), \quad N(1680), \quad N(1700), \quad N(1720)$, $N(1710), \quad \Delta(1232), \quad \Delta(1600), \quad \Delta(1620), \quad \Delta(1700)$, $\Delta(1905), \Delta(1910), \Delta(1920)$, and $\Delta(1950) . W_{\text {cut }}$ is a free parameter that determines the end of the shallow inelastic scattering (SIS) region. The nominal value is set to $W_{\text {cut }}=1.7 \mathrm{GeV} / c^{2}$.

In the version of GENIE used in this work, there is the option to select one of several neutrino-induced resonance production calculations performed by Rein and Sehgal [5], Kuzmin, Lyubushkin, and Naumov [6,7], and Berger and Sehgal [8]. The last two models are extensions of the first one, that account for nonzero lepton masses. Both models are based on the same formalism and the only difference between them is that the latter includes the pion-pole contribution to the hadronic axial current. The term $d^{2} \sigma^{\mathrm{DIS}} / d Q^{2} d W$ represents the GENIE calculation of the deep inelastic scattering (DIS) cross section that, in all relevant GENIE comprehensive model configurations, is carried out using an effective leading-order model with the modifications suggested by Bodek and Yang [9] to describe scattering at low momentum-transfers. This model is the foundation of both the DIS model and the SIS model in GENIE.

The term $d^{2} \sigma^{\text {SIS }} / d Q^{2} d W$ requires some elaboration. It represents the cross-section contribution from nonresonance shallow inelastic scattering in the resonance region. In GENIE, this cross section is computed with an empirical model where the Bodeck and Yang inclusive deep-inelastic cross section is extrapolated into the resonance region and it is decomposed, via the GENIE AGKY [10] hadronization model, into the cross sections for different hadronic multiplicity channels. The extrapolation of the DIS model down to the inelastic threshold, $W<W_{\text {cut }}$, includes, on average, the effect of the resonances [11]. Notice that, even though the Bodeck and Yang model is capable of describing the inclusive cross section at the inelastic threshold, we prefer to utilize an explicit resonance model. The contribution for hadronic multiplicities two and three, that are responsible for producing many final states similar to those produced via resonance excitation, are tuned to remove double counting. This tuning is the main topic of this work.

The nonresonance SIS cross section can be written as

$$
\frac{d^{2} \sigma^{\mathrm{SIS}}}{d Q^{2} d W}=\frac{d^{2} \tilde{\sigma}^{\mathrm{DIS}}}{d Q^{2} d W} \cdot \Theta\left(W_{\mathrm{cut}}-W\right) \cdot \sum_{m} f_{m}\left(Q^{2}, W\right),
$$

where $\tilde{\sigma}^{\mathrm{DIS}}$ represents the extrapolated deep-inelastic cross section into the resonance region, and $m$ refers to the multiplicity of the hadronic system. The factor $f_{m}$ relates the total calculated DIS cross section to the DIS contribution to this particular multiplicity channel. These factors are computed as

$$
f_{m}\left(Q^{2}, W\right)=R_{m} P_{m}^{\mathrm{had}}\left(Q^{2}, W\right)
$$

where $R_{m}$ is an adjustable parameter and $P_{m}^{\text {had }}$ is the probability, taken from the GENIE hadronization model, that the DIS final state hadronic system multiplicity would be equal to $m$.

At the low- $W$ values relevant for SIS, $P_{m}^{\text {had }}$ is constructed using the low-mass empirical AGKY model [10]. By construction, its distribution has an average $\langle m\rangle$ that increases logarithmically with $\mathrm{W}$,

$$
\begin{aligned}
\langle m\rangle\left(Q^{2}, W\right)= & \alpha+\beta \ln \left(\frac{W^{2}}{\mathrm{GeV}^{2} / \mathrm{c}^{4}}\right) \\
& +\beta^{\prime} \ln \left(\frac{Q^{2}}{\mathrm{GeV}^{2} / \mathrm{c}^{2}}\right)
\end{aligned}
$$

and the whole distribution is constructed from the average according to

$$
P_{m}^{\mathrm{had}}\left(Q^{2}, W\right)=\frac{1}{\langle m\rangle} \psi\left(\frac{m}{\langle m\rangle}\right),
$$

where the function $\psi$ (the Levy function) has the following form

$$
\psi(z)=\frac{2 e^{-c} c^{c z+1}}{\Gamma(c z+1)}, \quad z \equiv \frac{m}{\langle m\rangle} .
$$

In the above expressions, $\alpha, \beta, \beta^{\prime}$, and $c$ are adjustable parameters. In principle, $\alpha, \beta, \beta^{\prime} c$, and $R_{m}$, are different for each initial state $(\nu+p, \nu+n, \bar{\nu}+p, \bar{\nu}+n)$ and are different for charged current and neutral current interactions. A new tune of the neutrino-induced hadronization models in GENIE is currently in progress and, in future, it may be possible to perform a joint tuning of the GENIE cross section and hadronization modeling components for barenucleon targets. However, at this present work, the parameters $\alpha, \beta, \beta^{\prime}$, and $c$ were kept at the default values of the AGKY model in GENIE v3. For easy reference, the relevant values for the channels studied in this work are included in Table I. No dependence on $Q^{2}$ has been observed in $\nu$ and $\bar{\nu}$ scattering data [12], hence $\beta^{\prime}=0$ for all channels.

For most inelastic processes simulated in neutrinonucleus scattering by all current GENIE comprehensive model configurations, the total inelastic differential cross section for scattering off bare nucleons takes center stage. In Fig. 1, the contribution to the $\nu_{\mu}$ charged current (CC) and $\bar{\nu}_{\mu} \mathrm{CC}$ inclusive cross sections on isoscalar targets in 
TABLE I. Relevant default GENIE v3 AGKY parameters for $\nu_{\mu}$ and $\bar{\nu}_{\mu} \mathrm{CC}$ interactions on proton and neutron. The parameter values are extracted from [13] neutrinos and from [14] for antineutrinos.

\begin{tabular}{lcccc}
\hline \hline & \multicolumn{4}{c}{ Initial state } \\
\cline { 2 - 5 } Parameter & $\nu_{\mu} p$ & $\nu_{\mu} n$ & $\bar{\nu}_{\mu} p$ & $\bar{\nu}_{\mu} n$ \\
\hline$\alpha$ & 0.40 & -0.20 & 0.02 & 0.80 \\
$\beta$ & 1.42 & 1.42 & 1.28 & 0.95 \\
$c$ & 7.93 & 5.22 & 5.22 & 7.93 \\
\hline \hline
\end{tabular}

GENIE is shown for the different interaction processes. The CC RES and SIS/DIS CC cross-section contribution for different neutrino energies is shown in Fig. 2.

\section{COMPREHENSIVE MODEL CONFIGURATIONS IN GENIE V3}

GENIE has a large degree of configuration; for each process (RES, DIS, etc.) the system offers a number of alternative models to be used for event generation. In previous GENIE releases, only one model-process

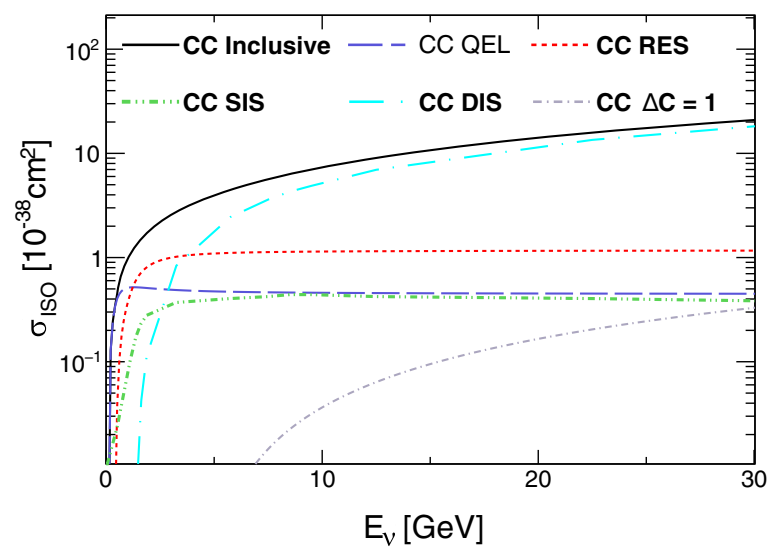

(a) $\nu_{\mu}$ CC on isoscalar targets.

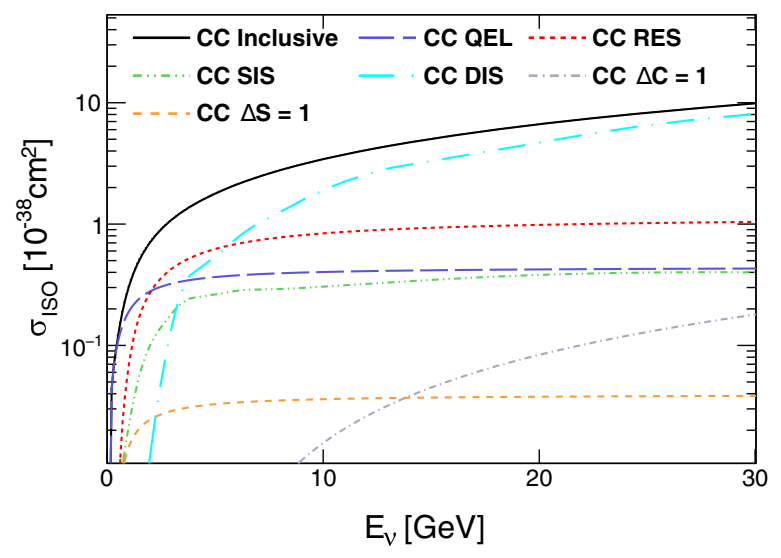

(b) $\bar{\nu}_{\mu}$ CC on isoscalar targets.

FIG. 1. Summary of neutrino and antineutrino cross sections on isoscalar targets. mapping was suggested by the out of the box configuration, despite the availability of alternative models. Yet, there was no guidance on how to correctly use different configurations according to the author and developers. In fact, the processes are not universal and their definitions are generator dependent. Hence, it was easy to come up with inconsistencies between the model configuration for different processes that were not supposed to be used together to get a correct comprehensive physics simulation.

This issue was addressed in GENIE v3 by introducing the concept of comprehensive model configuration (CMC) that is a consistent process-model association. Considering that GENIE already has about 20 different processes only for neutrinos, CMC definitions are quite complex objects and they need to be effectively named so that the community can use them unambiguously. For this purpose, the Collaboration developed a specific naming convention discussed in Appendix A. Sec. III A describes the models used in CMCs relevant for neutrino interactions.

\section{A. CMCs available in GENIE v3}

Several CMCs are available in GENIE v3, but they can be grouped together as their scopes are common. The first group of CMCs is historically motivated; it is based on the default configuration and simply provides updates for processes that were introduced later. The second family is an improvement of the first group in terms of the resonance model. The third one was constructed aiming to deliver the most up to date theoretical nuclear matter simulations. Out of these main ideas, a number of CMCs can be constructed by simply changing minor aspects like final state interaction (FSI) modeling or form factors. Here, we briefly summarize the modeling components used in each comprehensive model configuration available in GENIE v3 that are relevant for this paper. More are available in Appendix B. An extreme summary of the CMC model content is presented in Table II.

\section{G18_01a, G18_01b, G18_01c, and G18_01d}

These comprehensive models share an identical crosssection model construction, which is an adiabatic update of the historical default cross-section model of GENIE v2, now named as G00_00a CMC. For interactions on nucleons and nuclei, it relies on implementations of the following models: the Ahrens model [19] for neutral current (NC) elastic, the Llewellyn Smith model [17] for CC quasielastic, the Rein-Sehgal model [5] for NC and CC resonance production, the Rein-Sehgal model [18] for NC and CC coherent pion production, the Bodek-Yang model [9] for $\mathrm{NC}$ and $\mathrm{CC}$ deep inelastic scattering and nonresonance shallow inelastic scattering, the Kovalenko model [20] for quasielastic charm production, and the Aivazis-OlnessTung slow rescaling model [21] for deep inelastic charm production. Nuclear cross sections are calculated within the framework of a relativistic Fermi gas model, following the 


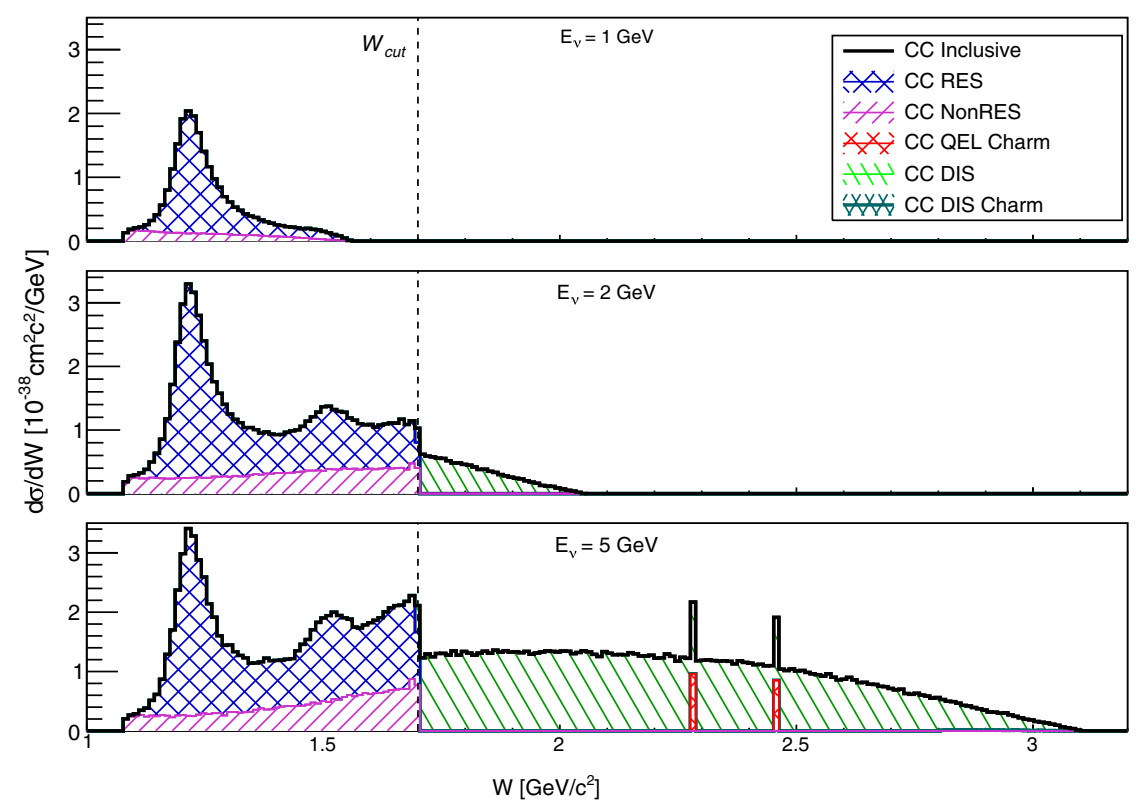

FIG. 2. RES and SIS/DIS differential cross section as a function of the invariant mass for three different neutrino energies using a $1 / E$ like flux. $W_{\text {cut }}$ divides the SIS and the DIS regions.

approach of Bodek-Ritchie [15]. Multinucleon processes in neutrino scattering off nuclear targets can be optionally enabled and simulated via an empirical GENIE model [1]. In addition, in GENIE v3, the adiabatic upgrade of the historical comprehensive model includes the simulation of processes that, previously, were either optional or missing. This includes both diffractive pion production based on an implementation of the Rein model [22], and quasielastic $|\Delta S|=1$ hyperon $\left(\Lambda^{0}, \Sigma^{-}, \Sigma^{0}\right)$ production based on the Pais model [23]. Single kaon production, although optionally available for neutrinos in GENIE v3 [24], is not yet available for antineutrinos and inclusion in any published GENIE comprehensive configurations was postponed until an antineutrino implementation is available and the kaon content of hadronic showers produced by GENIE has been retuned following the addition of the single-kaon generator. Both G18_01a and G18_01b comprehensive models employ a revised resonance-decay algorithm and an implementation of the AGKY [10] hadronization model that is unchanged with respect to that used at the latest releases of GENIE v2 series. Four comprehensive model variations are constructed by attaching different intranuclear hadron transport models to the same underlying cross section and hadronization models [25]. G18_01a uses an updated
INTRANUKE hA effective intranuclear rescattering model which is unique to GENIE, G18_01b uses the new INTRANUKE $\mathrm{hN}$ model implementing a full intranuclear cascade including medium corrections, G18_01c uses an interface to the GEANT4 [26] Bertini intranuclear cascade [27] (version 4.10.2) and G18_01d uses an interface to the INCL++ (version 5.2.9.5) implementation of the Liège intranuclear model [28].

\section{G18_02a, G18_02b, G18_02c, and G18_02d}

This is family of empirical models which is an evolved version of the G18_01[a-] ones. The general construction of the cross-section model is similar to the one discussed above, with the exception that the implementations of the Rein-Sehgal models for CC and NC resonance neutrino-production, as well as for $\mathrm{CC}$ and $\mathrm{NC}$ coherent production of mesons, were replaced with updated models by Berger-Sehgal [8]. Similarly to G18_01[a-d], four comprehensive model variations are constructed by using alternative intranuclear hadron transport models on top of the same underlying cross section and hadronization models (a) INTRANUKE/hA, (b) INTRANUKE/hN, (c) GEANT4/Bertini, and (d) INCL ++ ).

TABLE II. List of relevant models for (anti)neutrino CC interactions on free nucleons.

\begin{tabular}{lcc}
\hline \hline & \multicolumn{2}{c}{ CMC } \\
\cline { 2 - 3 } Model & G18_01* G18_02* & G18_10* \\
\hline Nuclear model & Relativistic Fermi gas [15] & Local Fermi gas [16] \\
CC QEL & Llewellyn Smith model [17] & Valencia model [16] \\
CC RES & Rein-Sehgal [18] & Bodek-Yang [9] \\
CC DIS & & Berger-Sehgal [8] \\
\hline \hline
\end{tabular}




\section{3. $G 18 \_10 a, G 18 \_10 b, G 18 \_10 c$, and $G 18 \_10 d$}

This is a family of models derived from the improved empirical ones (G18_02[a-d]) described above, by substituting both the Llewellyn Smith CC quasielastic model [17] and GENIEs empirical multinucleon model with implementations of the corresponding Valencia models by Nieves et al. [16]. This family of comprehensive models provides a firmer theoretical basis for the simulation of neutrino-nucleus scattering around the quasielastic peak. Within this family of models, the nuclear environment is modeled using a local Fermi gas, matching the inputs used for the published Valencia calculations. Again, four comprehensive model variations [(a)-(d)] are constructed by using alternative intranuclear hadron transport models, following the same naming convention introduced above. The implementation of the Valencia model in GENIE does not predict the kinematics of the outgoing hadrons and its description needs to be accompanied by one of the FSI models available in GENIE [(a)-(d)] [29].

\section{B. Free nucleons and CMCs}

Although a large number (16) of CMCs were summarized above, with respect to the cross sections for (anti)neutrino scattering off bare nucleons, there are only two different model constructions: The one used in (a) G18_01[a-d], and the one used in (b) G18_02[a-d], G18_10[a-d] and G18_10 $[i-1]$. The main difference between these two model constructions resides mainly in the treatment of the lepton mass. Although some differences can be expected between G18_10[a-d] and G18_10[i-1], because of different choices in the modeling of the axial form factor for quasielastic scattering, they do not manifest themselves in the context of this particular analysis.

Several variations of the tuning procedure were run and evaluated for testing purposes before converging to the procedure presented in this paper. Preliminary versions of this work were released in the GENIE v3 series (v3.0.0v3.0.6) in a series of tunes carrying the 02_11a label. The final results presented in the paper will be made available in GENIE v3.2 in a series of 16 tunes, one for each of the 16 comprehensive model configurations summarized above, labeled as 02_11b. For example, the tune G18_10a_02_11b corresponds to the G18_10a comprehensive model with the parameters determined through the tuning procedure discussed in this paper (02_11b). The GENIE tune naming convention is discussed in Appendix A. A full list of GENIE tunes is maintained in http://tunes.genie-mc.org. The preliminary versions (02_11a) of the tunes will be kept in GENIE v3.2, but they will be phased out in subsequent minor releases. It is useful to mention that CMCs whose parameters are not tuned using the procedures similar to those described in this paper are identified with the tune label 00_000, e.g., the configuration of G18_10a_02_11b before the tune is identified as G18_10a_00_000.

\section{DATA AND MODEL UNCERTAINTIES REVIEW}

The data used in this analysis are old and a careful review of the past analysis procedure is required in order to combine all the data together in a global analysis. This section summarizes the data details and how the models used in the fit behave in the same energy region.

\section{A. Datasets included in the fit and their systematics}

In the current work, we consider hydrogen and deuterium data from the ANL 12FT, BNL 7FT, FNAL 15FT, and BEBC bubble chamber experiments. The data represent integrated cross sections for different incoming neutrino energy bins for

(a) $\nu_{\mu}$ and $\bar{\nu}_{\mu}$ CC inclusive scattering [30-57].

(b) $\nu_{\mu}$ and $\bar{\nu}_{\mu}$ CC quasielastic scattering [30,40,54,58-66].

(c) $\nu_{\mu}$ and $\bar{\nu}_{\mu}$ CC single-pion production [59,67-75].

$\nu_{\mu}+n \rightarrow \mu^{-}+n+\pi^{+}$
$\nu_{\mu}+p \rightarrow \mu^{-}+p+\pi^{+}$
$\nu_{\mu}+n \rightarrow \mu^{-}+p+\pi^{0}$
$\bar{\nu}_{\mu}+p \rightarrow \mu^{+}+p+\pi^{-}$
$\bar{\nu}_{\mu}+n \rightarrow \mu^{+}+n+\pi^{-}$

(d) $\nu_{\mu}$ CC two-pion production [76].

$\nu_{\mu}+p \rightarrow \mu^{-}+n+2 \pi^{+}$

$\nu_{\mu}+p \rightarrow \mu^{-}+p+\pi^{+}+\pi^{0}$

$\nu_{\mu}+p \rightarrow \mu^{-}+n+\pi^{+}+\pi^{-}$

Not all of the available historical data has been used for the fit, as some datasets were superseded or reanalyzed, as in the case of ANL 12FT and BNL 7FT datasets. The latest analysis are used. A detailed summary of the datasets used in the fit is shown in Table III and in Fig. 3. Some of the datasets included in the tune consider hydrogen-neon mixtures. The nuclear effects of the neon in the target mixture are shown to be negligible [77].

Low energy bins have a higher contribution to the $\chi^{2}$ due to energy smearing and lack of unfolding in measurements. Hence, data points with $E_{\nu}<0.5 \mathrm{GeV}$ are removed from the fit. In total, the tune is performed with 169 data points from bubble chamber experiments. Different analysis methods were implemented in each experiment, such as cuts applied on the $W$ invariant mass, the outgoing muon momentum, or the total longitudinal momentum of the final state. The associated GENIE prediction has been corrected by applying the same cuts to the generated events. Moreover, datasets from the same experiments are not independent as they share the same neutrino flux, detector, analysis methodology, etc. Although it is clear that some correlated uncertainties exist, the data releases do not contain any information about the correlation between them. In the GENIE database we added a systematic error to the datasets of $15 \%$. The methodology used to include them in the fit is detailed in Sec. V C. Other free nucleon data on heavier targets are available but used only for comparison with the GENIE prediction. No correction for nuclear effects is considered for deuterium targets. 
TABLE III. A summary of cross-section data used in this work. The number of data points released by each analysis $\left(N_{p}\right)$, the neutrino energy range covered $E_{\nu}$, the type of target and the cuts applied in the analysis procedure are specified in the table.

\begin{tabular}{|c|c|c|c|c|c|}
\hline Experiment & $N_{p}$ & Energy $[\mathrm{GeV}]$ & Target & Cuts & References \\
\hline \multicolumn{6}{|c|}{$\nu_{\mu}+N \rightarrow \mu^{-} X$} \\
\hline BNL 7FT & 13 & $0.6-10$ & ${ }^{2} \mathrm{H}$ & & {$[45]$} \\
\hline BEBC & 3 & $10-50$ & ${ }^{1} \mathrm{H}, \mathrm{Ne}$ & & [43] \\
\hline \multirow[t]{2}{*}{ FNAL } & 6 & $10-110$ & ${ }^{2} \mathrm{H}$ & & {$[36]$} \\
\hline & 5 & $100-110$ & ${ }^{1} \mathrm{H}, \mathrm{Ne}$ & & [48] \\
\hline \multicolumn{6}{|c|}{$\bar{\nu}_{\mu}+N \rightarrow \mu^{+} X$} \\
\hline \multirow[t]{4}{*}{ BEBC } & 3 & $11-110$ & ${ }^{1} \mathrm{H}, \mathrm{Ne}$ & & {$[31]$} \\
\hline & 1 & $10-50$ & ${ }^{1} \mathrm{H}, \mathrm{Ne}$ & & [43] \\
\hline & 6 & $30-110$ & ${ }^{1} \mathrm{H}, \mathrm{Ne}$ & & {$[32]$} \\
\hline & 1 & $10-110$ & ${ }^{1} \mathrm{H}, \mathrm{Ne}$ & & [44] \\
\hline BNL 7FT & 1 & $1-4$ & ${ }^{1} \mathrm{H}$ & & {$[54]$} \\
\hline \multirow[t]{2}{*}{ FNAL } & 5 & $10-110$ & ${ }^{2} \mathrm{H}, \mathrm{Ne}$ & & {$[50]$} \\
\hline & 7 & $10-80$ & ${ }^{2} \mathrm{H}, \mathrm{Ne}$ & & {$[55]$} \\
\hline \multicolumn{6}{|c|}{$\nu_{\mu} n \rightarrow \mu^{-} n \pi^{+}$} \\
\hline ANL 12FT & 5 & $0.3-2$ & ${ }^{1} \mathrm{H},{ }^{2} \mathrm{H}$ & & {$[68]$} \\
\hline ANL 12FT,ReAna & 7 & $0.3-3$ & ${ }^{2} \mathrm{H}$ & & [69] \\
\hline BNL 7FT,ReAna & 11 & $0.1-4$ & ${ }^{2} \mathrm{H}$ & & [69] \\
\hline \multicolumn{6}{|c|}{$\nu_{\mu} p \rightarrow \mu^{-} p \pi^{+}$} \\
\hline ANL 12FT,ReAna & 8 & $0-1.6$ & ${ }^{2} \mathrm{H}$ & & [69] \\
\hline BNL 7FT,ReAna & 7 & $0-7$ & ${ }^{2} \mathrm{H}$ & & [69] \\
\hline \multirow[t]{3}{*}{ BEBC } & 7 & $1-30$ & ${ }^{1} \mathrm{H}$ & $W<1.4 \mathrm{GeV}$ & [71] \\
\hline & 6 & $5-100$ & ${ }^{2} \mathrm{H}$ & $W<2 \mathrm{GeV}$ & [59] \\
\hline & 5 & $10-80$ & ${ }^{1} \mathrm{H}$ & $W<2 \mathrm{GeV}$ & {$[73]$} \\
\hline FNAL & 3 & $10-30$ & ${ }^{1} \mathrm{H}$ & $W<1.4 \mathrm{GeV}$ & {$[78]$} \\
\hline \multicolumn{6}{|c|}{$\nu_{\mu} n \rightarrow \mu^{-} p \pi^{0}$} \\
\hline ANL 12FT & 5 & $0.2-2$ & ${ }^{2} \mathrm{H}$ & & {$[68]$} \\
\hline ANL 12FT,ReAna & 7 & $0.2-2$ & ${ }^{2} \mathrm{H}$ & & [69] \\
\hline BNL 7FT,ReAna & 10 & $0.4-3$ & ${ }^{2} \mathrm{H}$ & & [69] \\
\hline \multicolumn{6}{|c|}{$\nu_{\mu} p \rightarrow \mu^{-} n \pi^{+} \pi^{+}$} \\
\hline ANL 12FT & 5 & $1-6$ & ${ }^{2} \mathrm{H}$ & & [76] \\
\hline \multicolumn{6}{|c|}{$\nu_{\mu} p \rightarrow \mu^{-} p \pi^{+} \pi^{0}$} \\
\hline ANL 12FT & 5 & $1-6$ & ${ }^{2} \mathrm{H}$ & & {$[76]$} \\
\hline \multicolumn{6}{|c|}{$\nu_{\mu} n \rightarrow \mu^{-} p \pi^{+} \pi^{-}$} \\
\hline ANL 12FT & 5 & $8-6$ & ${ }^{2} \mathrm{H}$ & & {$[76]$} \\
\hline BNL 7FT & 10 & $0-20$ & ${ }^{2} \mathrm{H}$ & & {$[36]$} \\
\hline \multicolumn{6}{|c|}{$\bar{\nu}_{\mu} p \rightarrow \mu^{+} p \pi^{-}$} \\
\hline FNAL & 1 & $5-70$ & ${ }^{1} \mathrm{H}$ & $W<1.9 \mathrm{GeV}$ & {$[74]$} \\
\hline \multicolumn{6}{|c|}{$\nu_{\mu}+n \rightarrow \mu^{-}+p$} \\
\hline \multirow[t]{2}{*}{ ANL 12FT } & 7 & $0-2$ & ${ }^{2} \mathrm{H}$ & & {$[58]$} \\
\hline & 8 & $0-2$ & ${ }^{1} \mathrm{H},{ }^{2} \mathrm{H}$ & & {$[30]$} \\
\hline BNL 7FT & 4 & $0.2-2$ & ${ }^{2} \mathrm{H}$ & & {$[63]$} \\
\hline $\mathrm{BEBC}$ & 5 & $20-40$ & ${ }^{2} \mathrm{H}$ & & [59] \\
\hline FNAL & 2 & $0-50$ & ${ }^{2} \mathrm{H}$ & & {$[60]$} \\
\hline
\end{tabular}




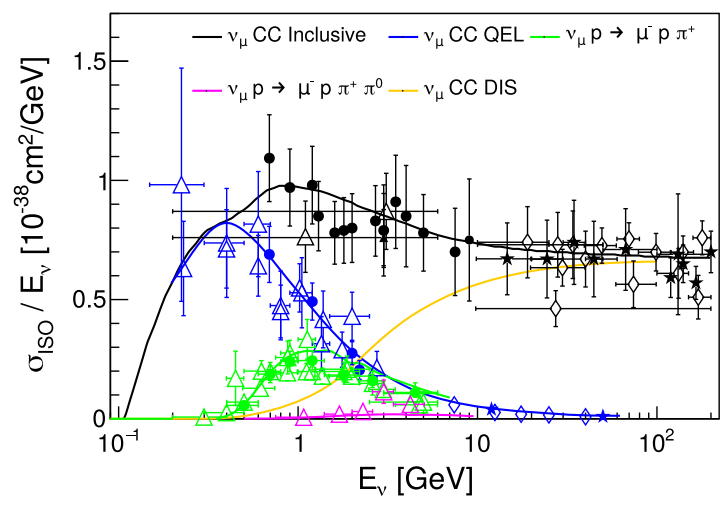

(a) $\nu_{\mu}$ CC cross section.

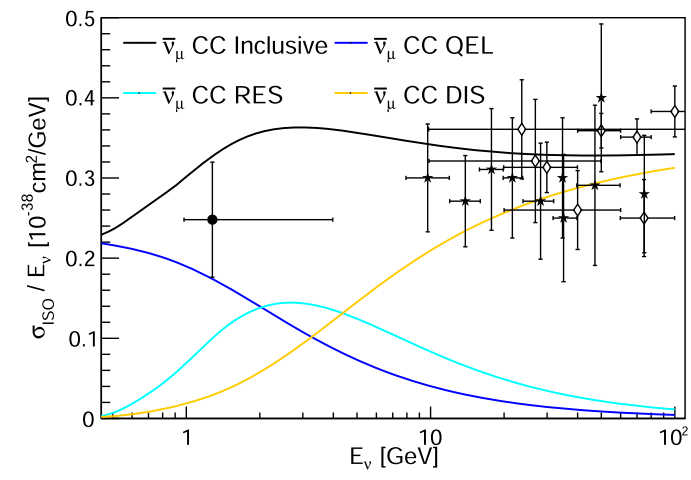

(b) $\bar{\nu}_{\mu}$ CC cross section.

FIG. 3. Charged current cross section on isoscalar targets as a function of the incoming neutrino energy. Breakdown of quasielastic, one- and two-pion production and deep inelastic processes is shown. The predictions are computed using the G18_02a_00_000 configuration. The data on hydrogen and deuterium targets from Table III is shown if available from ANL 12FT (triangle), BNL 7FT (filled circle), BEBC (diamond), and FNAL (star).

\section{B. Model uncertainties}

The SIS cross section is tuned within the CMCs using either the Rein-Sehgal or Berger-Sehgal resonance models, (see Sec. III A). The tuning main goal is the best value estimation for nine of the parameters that drive the GENIE predictions in the SIS region. These parameters are the $W_{\text {cut }}$ as defined in Eq. (1), the four $R_{m}$ coefficients for CC interactions on neutron/proton with $m=2,3$ from the SIS region Eq. (3), the axial masses used in the dipole form factors for RES and quasielastic (QE) interactions, and two global scaling factors, $S_{\text {RES }}$ for the RES cross section and $S_{\text {DIS }}$ for the DIS cross section. For clarity, we will refer to $R_{m}$ parameters with the number of pions in the final state, namely $R_{\nu p}^{\mathrm{CC} 1 \pi}, R_{\nu p}^{\mathrm{CC} 2 \pi}, R_{\nu n}^{\mathrm{CC} 1 \pi}$, and $R_{\nu n}^{\mathrm{CC} 2 \pi}$.

Most of the parameters described in the previous paragraph are empirical and only valid for GENIE. Therefore, there are no expectations about their values. The notable exceptions are the axial masses. For the G18_01a(/b/c/d) and $\mathrm{G} 18 \_02 \mathrm{a}(\mathrm{b} / \mathrm{c} / \mathrm{d})$ CMCs, the axial form factors are described using the dipole parametrization which is a function of the invariant transferred momentum $\left(Q^{2}\right)$

$$
F_{A}\left(Q^{2}\right)=F_{A}(0)\left(1+\frac{Q^{2}}{M_{A}^{2}}\right)^{-2}
$$

with $F_{A}(0)=g_{A}=-1.2695 \pm 0.002$ [79]. The axial mass, $M_{A}$, is extracted from data. There are different masses for both interaction types; $M_{A}^{\mathrm{QEL}}$ and $M_{A}^{\mathrm{RES}}$. Both of these are evaluated from neutrino data on deuterium targets. The latest world average values for the axial masses are

$$
\begin{aligned}
M_{A}^{\mathrm{QE}} & =1.014 \pm 0.014 \mathrm{GeV} / c^{2}, \\
M_{A}^{\mathrm{RES}} & =1.12 \pm 0.03 \mathrm{GeV} / c^{2} .
\end{aligned}
$$

The extraction of these parameters requires neutrino differential cross sections as a function of $Q^{2}$ that are not used in this analysis.

Table IV summarizes the parameter prefit values and the allowed ranges. Previous fits to data are taken into account for the determination of the ranges [80,81]. Each of the parameters have a different sensitivity to each dataset as different scattering mechanisms are involved. The response of each parameter in the inclusive and exclusive cross

TABLE IV. Parameters of interest of the tunes and their statistical properties as used in the fitting procedures. The Default values correspond to the nominal values from GENIE v2 [1].

\begin{tabular}{lccccc}
\hline \hline Parameter & GENIE parameter name & Default value & Min value & Max value & Prior \\
\hline$W_{\text {cut }}\left(\mathrm{GeV} / \mathrm{c}^{2}\right)$ & Wcut & 1.7 & 1.5 & 2.3 & $\ldots$ \\
$M_{A}^{\mathrm{QE}}\left(\mathrm{GeV} / \mathrm{c}^{2}\right)$ & QEL-Ma & 0.999 & 0.75 & 1.10 & $1.014 \pm 0.014[82]$ \\
$M_{A}^{\mathrm{RES}}\left(\mathrm{GeV} / \mathrm{c}^{2}\right)$ & RES-Ma & 1.12 & 0.8 & 1.3 & $1.12 \pm 0.03[80]$ \\
$R_{\nu p}^{\mathrm{CC} 1 \pi}$ & DIS-HMultWgt-vp-CC-m2 & 0.10 & 0.0 & 0.4 & $\ldots$ \\
$R_{\nu p}^{\mathrm{CC} 2 \pi}$ & DIS-HMultWgt-vp-CC-m3 & 1.00 & 0.0 & 2.0 & $\ldots$ \\
$R_{\nu n}^{\mathrm{CC} 1 \pi}$ & DIS-HMultWgt-vn-CC-m2 & 0.30 & 0.0 & 0.35 & $\ldots$ \\
$R_{\nu n}^{\mathrm{CC} 2 \pi}$ & DIS-HMultWgt-vn-CC-m3 & 1.00 & 0.8 & 3.0 & $\ldots$ \\
$S_{\mathrm{RES}}$ & RES-CC-XSecScale & 1.0 & 0.6 & 1.2 & $\ldots$ \\
$S_{\mathrm{DIS}}$ & DIS-CC-XSecScale & 1.032 & 0.9 & 1.15 & $1 \pm 0.05$ \\
\hline
\end{tabular}


sections is studied by varying each of them independently within the studied range. In Fig. 4, each parameter response is shown for inclusive and exclusive cross sections. When more than one parameter in the plot is impacting the same cross section, i.e., $\mathrm{CC}$ inclusive, the variations are added in quadrature.

At the Monte Carlo level, where no correlation between the parameters is considered, the impact of each of the parameters in the cross section can be classified as influencing a variation on

(1) The CC quasielastic cross section.

(2) The CC RES cross section.

(3) The CC DIS cross section.

For instance, $M_{A}^{\mathrm{QE}}$ will only affect the quasielastic crosssection prediction, as summarized in Fig. 4(a). Notice though that, at the tune level, this will no longer hold as the introduction of flux-nuisance parameters correlates exclusive channels. Hence, this will introduce a correlation between $M_{A}^{\mathrm{QE}}$ and the SIS parameters.

The description of the CC RES cross section will be affected by the RES axial mass $M_{A}^{\text {RES }}$ [Fig. 4(a)], the resonance scaling parameter $S_{\text {RES }}$ [Fig. 4(b)], and $W_{\text {cut }}$
[Fig. 4(d)]. The G18_01a_00_000 and G18_02a_00_000 configurations overestimate one-pion production processes, and would favor a reduction in the CC RES cross section. Variations of $M_{A}^{\mathrm{RES}}$ have a huge impact on both exclusive and inclusive CC cross sections in the few-GeV region. However, as it is explained in Sec. VC, this parameter should agree with the world average extracted from fits to the axial form factor [80] and a deviation from this result is disfavored by previous fits to data. Consequently, a reduction of $S_{\text {RES }}$ is expected to improve the agreement with one-pion production data. On the other hand, $W_{\text {cut }}$ will play an important role as it determines the number of resonances included in the CC RES calculation. The current default, $W_{\text {cut }}=1.7 \mathrm{GeV} / c^{2}$, discards the resonances contributed at $W>W_{\text {cut }}$. Therefore, an increase on $W_{\text {cut }}$ will incorporate new resonances in the calculation that were not taken into account in previous tunes. This increase is favored by two-pion production data, as heavier resonances producing more than one pion are incorporated.

The SIS region is treated by combining two cross-section models, one for DIS and one for RES interactions. Thus, in

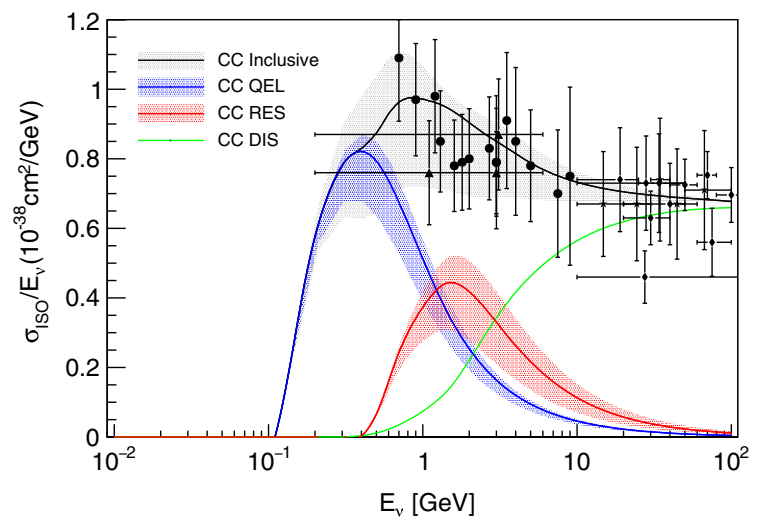

(a) $M_{A}^{\mathrm{RES}}$ and $M_{A}^{\mathrm{QE}}$ impact.

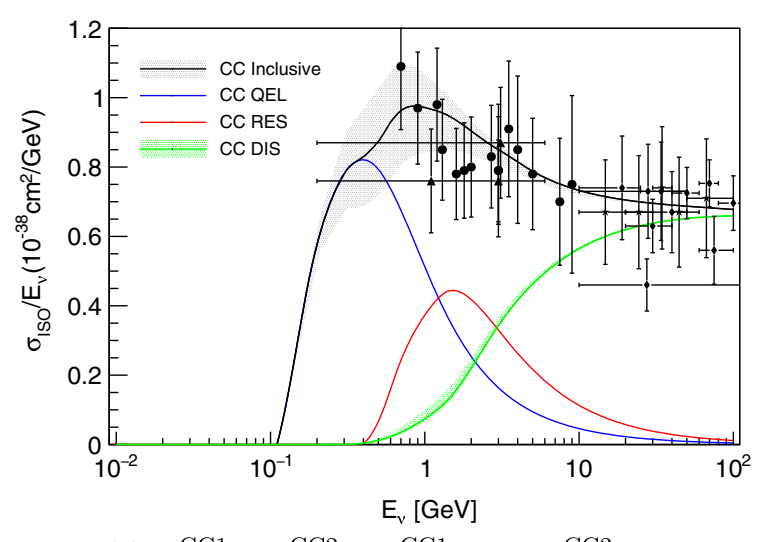

(c) $R_{\nu p}^{\mathrm{CC} 1 \pi}, R_{\nu p}^{\mathrm{CC} 2 \pi}, R_{\nu n}^{\mathrm{CC} 1 \pi}$ and $R_{\nu n}^{\mathrm{CC} 2 \pi}$ impact.

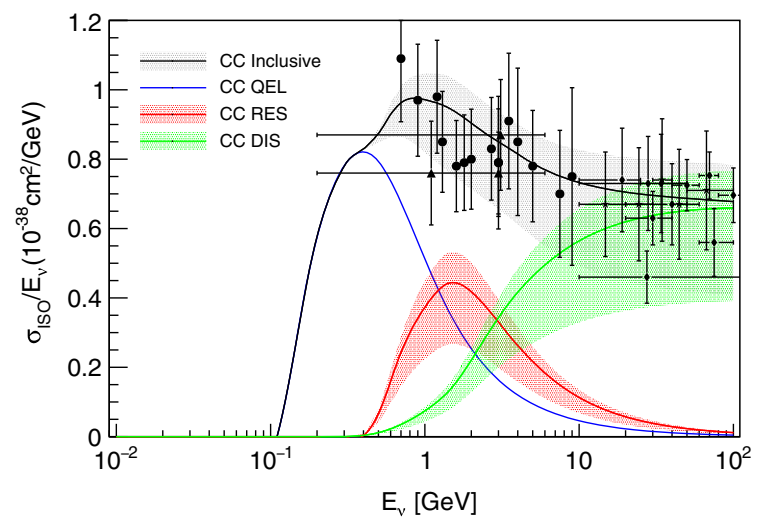

(b) $S_{\mathrm{RES}}$ and $S_{\mathrm{DIS}}$ impact.

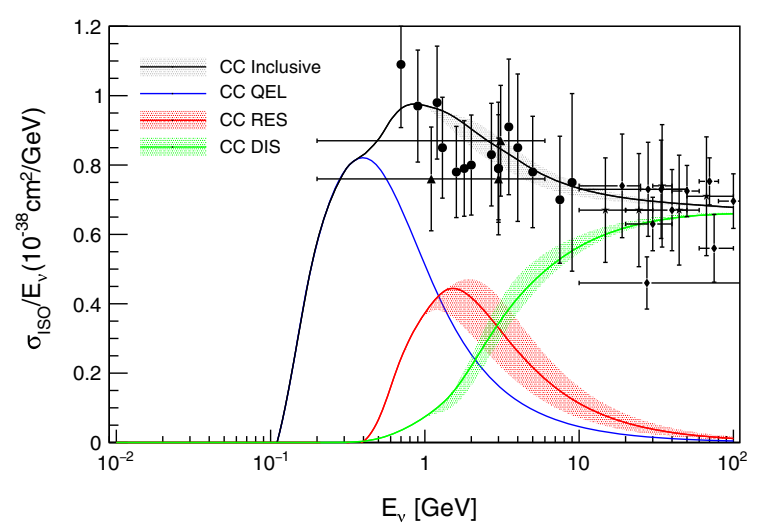

(d) $W_{\text {cut }}$ impact.

FIG. 4. $\quad \nu_{\mu}$ CC inclusive G18_02a prediction against hydrogen and deuterium data. Each parameter response is characterized within the tuned region, specified in Table IV. Data corresponding to hydrogen and deuterium targets from ANL 12FT (triangle), BNL 7FT (filled circle), BEBC (diamond), and FNAL 15FT (star). 
that region, many parameters have a visible effect on the predictions as can be seen in Fig. 4; regardless of the parameter considered in the plot there is always a visible error band in the few-GeV region. This is a clear hint for the presence of degeneracy that must be faced by our global tunes. An example of this is given by the $R_{m}$ and the $S_{\text {DIS }}$ parameters, which act as scaling factors for the DIS contribution at $W<W$ cut. As mentioned above, a desired result of the tune is to reduce the one-pion prediction and increase the two-pion production. This can be accomplished via alterations of either the $R_{n}$ and/or the $S_{\text {DIS }}$ parameters.

\section{BARE-NUCLEON CROSS-SECTION TUNING PROCEDURE}

This section describes the core ideas behind the paper. Most of these are not specific for this work; they are general concepts developed within the GENIE tuning system and can therefore apply to future tune releases.

\section{A. Likelihood construction}

The GENIE integrated cross-section prediction is denoted with $\sigma_{\mathrm{th}}^{i}\left(E_{k} \mid \boldsymbol{\theta}\right)$, where $E_{k}$ is the neutrino energy, $\boldsymbol{\theta}$ is a vector [83] of the adjustable physics parameters introduced in Sec. IV B, and $i$ is any of the ten reaction processes considered in the work presented in Table III. Using $\sigma_{\text {th }}^{i}(E \mid \boldsymbol{\theta})$, we produce the corresponding prediction for the $k$ th energy bin of the $j$ th dataset for the $i$ th reaction type,

$$
\sigma_{\mathrm{th}}^{i j}\left(E_{k} \mid \boldsymbol{\theta}\right)=\varepsilon^{i j}\left(E_{k}, \boldsymbol{\theta}\right) \sigma_{\mathrm{th}}^{i}\left(E_{k} \mid \boldsymbol{\theta}\right)
$$

where $\varepsilon^{i j}\left(E_{k}, \boldsymbol{\theta}\right)$ are dataset-dependent efficiencies expressing the fraction of events from the $i$ th process that survive the kinematical cuts imposed by the experiment (see Table III). The statistical error due to the Monte Carlo (MC) sample size is also evaluated and this is denoted $\delta \sigma^{i j}\left(E_{k} \mid \boldsymbol{\theta}\right)$.

Performing a multiparameter brute-force scan and tune using $\sigma_{\mathrm{th}}^{i j}\left(E_{k} \mid \boldsymbol{\theta}\right)$ is computationally inefficient. As was highlighted in the introduction, the GENIE global analysis framework relies on Professor [2] to reduce the computational complexity of brute-force scans while allowing for massive parallelization. Using the values of $\sigma_{\mathrm{th}}^{i j}\left(E_{k} \mid \boldsymbol{\theta}\right)$ computed for a number $\left(N_{R}\right)$ of randomized $P$-dimensional vectors $\boldsymbol{\theta}$, produced within the $P$-dimensional hypercube defined by the parameter ranges given in Table IV, we use Professor to generate a parametrization of $\sigma_{\mathrm{th}}^{i j}\left(E_{k} \mid \boldsymbol{\theta}\right)$ and $\delta \sigma^{i j}\left(E_{k} \mid \boldsymbol{\theta}\right)$ that will be denoted with $\tilde{\sigma}_{\mathrm{th}}^{i j}\left(E_{k} \mid \boldsymbol{\theta}\right)$ and $\delta \tilde{\sigma}^{i j}\left(E_{k} \mid \boldsymbol{\theta}\right)$ respectively. As discussed in [2], the parametrization is a generic polynomial of order $M$ in the $P$-dimensional space, whose analytical form is

$$
\begin{aligned}
\tilde{\sigma}_{\text {th }}^{i j}\left(E_{k} \mid \boldsymbol{\theta}\right)= & \alpha_{0}^{i j k}+\sum_{n=1}^{P} \beta_{n}^{i j k} \theta_{n}+\sum_{n \leq m} \gamma_{n m}^{i j k} \theta_{n} \theta_{m} \\
& +\cdots+\sum_{n_{1} \leq \ldots \leq n_{M}} \xi_{n_{1} \ldots n_{M}}^{i j k} \prod_{\ell=1}^{M} \theta_{n_{\ell}}
\end{aligned}
$$

where $\theta_{n}$ is the coordinate of the $n$th parameter. The polynomial order $M$ is set by the user. The coefficients $\alpha_{0}^{i j k}, \beta_{n}^{i j k}, \gamma_{(n m)}^{i j k}, \ldots, \xi_{\left(n_{1} \ldots n_{M}\right)}^{i j k}$ are determined by Professor fitting the parametrization against the computed $\sigma_{\text {th }}^{i j}\left(E_{k} \mid \boldsymbol{\theta}\right)$. In the analysis presented here, a fourth-order polynomial was used for the G18_01a comprehensive model configuration while a fifth-order polynomial was used for G18_02a. Particularly, $N_{R}=1500$ for G18_01a and $N_{R}=$ 2183 for G18_02a. The accuracy of the parametrization is demonstrated in the residual distributions shown in Fig. 5. (fourth-order polynomials were the initial targets for both G18_01a and G18_02a fits). Some boundaries were relaxed during the analysis and more scan points were added allowing the usage of fifth-order polynomials for G18_02a. The parametrization $\tilde{\sigma}_{\mathrm{th}}^{i j}\left(E_{k} \mid \boldsymbol{\theta}\right)$ is used instead of
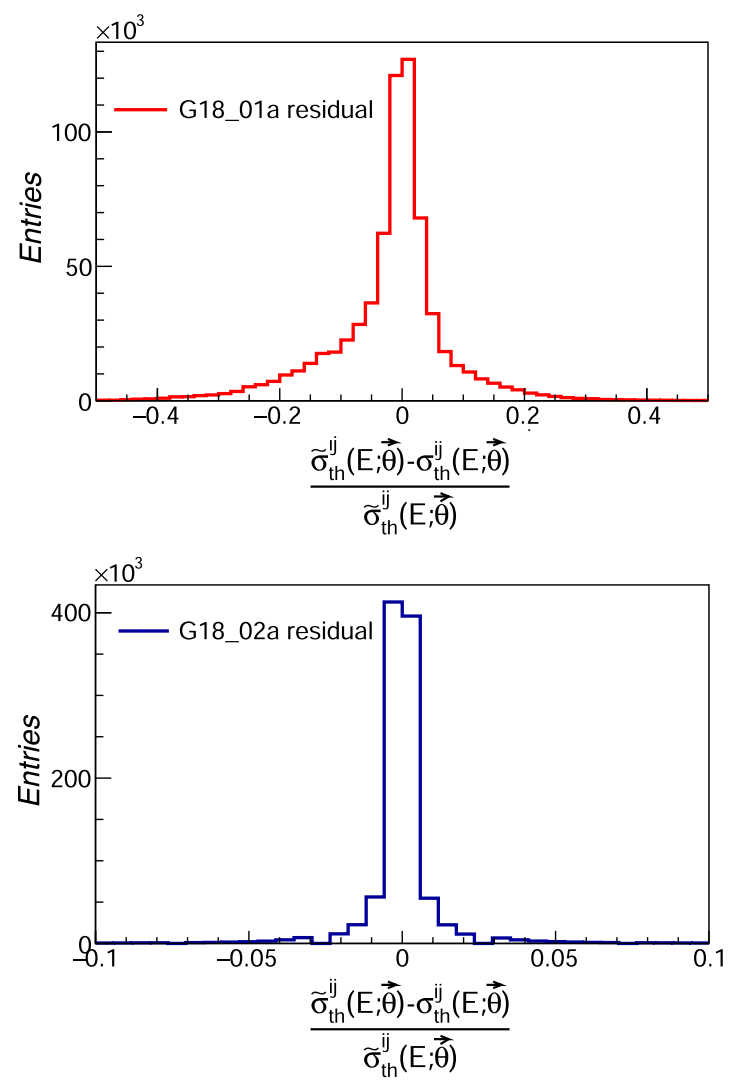

FIG. 5. Fractional difference between true Monte Carlo predictions calculated with a given $\vec{\theta}$ set vs the Professor parametrization for both tunes. The G18_02a(b) parametrization is improved as the number of scan points used and polynomial order $M$ are higher. 
the exact predictions in order to to estimate the best-fit parameters by minimizing the $\chi^{2}$.

\section{B. Treatment of systematic uncertainties}

A number of nuisance parameters, each with a corresponding prior, can also be used to tackle the problem of correlated datasets. As seen in Sec. IV A, there are different datasets coming from the same experiments (ANL 12FT, BNL 7FT, BEBC, and FNAL 15FT). Each of these experiments share the same flux (from either a neutrino or an antineutrino beam), analysis procedure, etc. Therefore, there is a correlation between the datasets, even though it has not been quantified in the data releases. A possible approach is to add nuisance parameters that can connect datasets from experiments that used the same neutrino beam [84]. As the main systematic uncertainty comes from the fluxes, the nuisance parameters will act as scaling factors for our predictions $\left(\tilde{\sigma}_{\mathrm{th}}^{i j}\left(E_{k} \mid \boldsymbol{\theta}\right)\right)$ and are same for datasets sharing the same flux.

Some of the ANL 12FT and BNL 7FT data were already corrected for the flux normalization [69]. Due to this correction, the associated systematic error is smaller and, accordingly, a more restricted nuisance parameter is applied to the reanalyzed datasets. These restricted parameters take into account other common systematics like reconstruction procedures, so they multiply all the predictions related to the same experiment. At the end of this procedure, each prediction can be scaled by up to two nuisance parameters, one for the flux and one for the remaining systematics. Thus, a total of nine independent nuisance parameters are used to account for the correlation between datasets. They are all the available combinations of experiment and neutrino flux exposure $\left(\nu_{\mu}\right.$ and $\left.\bar{\nu}_{\mu}\right)$ plus the restricted parameters for reanalyzed data,

$$
\begin{aligned}
\boldsymbol{f}= & \left(f^{\mathrm{ANL}}\left(\nu_{\mu}\right), f_{\mathrm{Re}}^{\mathrm{ANL}}\left(\nu_{\mu}\right), f^{\mathrm{BNL}}\left(\nu_{\mu}\right),\right. \\
& f_{\operatorname{Re}}^{\mathrm{BNL}}\left(\nu_{\mu}\right), f^{\mathrm{BNL}}\left(\bar{\nu}_{\mu}\right), f^{\mathrm{BEBC}}\left(\nu_{\mu}\right), \\
& \left.f^{\mathrm{BEBC}}\left(\bar{\nu}_{\mu}\right), f^{\mathrm{FNAL}}\left(\nu_{\mu}\right), f^{\mathrm{FNAL}}\left(\bar{\nu}_{\mu}\right)\right) .
\end{aligned}
$$

Quasielastic data for hydrogen and deuterium targets is included in the tune in order to constrain the nuisance parameters. Even though quasielastic data is not directly constraining the SIS parameters, it plays an important role to further constrain the fluxes of each experiment, as it is known at the $15 \%$ level.

The main advantage of this method is the unbiased choice of the nuisance parameters, as their values will be determined by the minimization of the likelihood function. For the calculation of best-fit points and the calculation of intervals, these nuisance parameters are profiled (on every instance of our fit they are eliminated by substituting them with the value that minimizes $\chi^{2}$ ).

\section{Discussion of priors}

The likelihood is corrected using priors on parameters of interest $(\boldsymbol{\theta})$ and nuisance parameters $(\boldsymbol{f})$. Priors allow us to incorporate in this analysis the appropriate prefit uncertainties and correlations for the parameters of interest. Only Gaussian priors are considered at present.

The priors applied to each nuisance parameter $f_{j}$ have a peak at 1 and different standard deviations $\delta f_{j}$. In general the total scaling factor applied to nonreanalyzed datasets are constrained by a conservative $15 \%$ of Gaussian prior, except for those nuisance parameters that act on the same experiment. Thus, the BEBC and FNAL 15FT experiments have only one associated scaling factor $\delta f^{\mathrm{BEBC}}=$ $\delta f^{\mathrm{FNAL}}=0.15$ for both neutrino and antineutrino fluxes; the same is true for $f^{\mathrm{BNL}}\left(\bar{\nu}_{\mu}\right)$. Up to two nuisance parameters can be applied to ANL 12FT and BNL 7FT data [i.e., $f^{A N L}\left(\nu_{\mu}\right)$ and $f_{\operatorname{Re}}^{A N L}\left(\nu_{\mu}\right)$ ]. The ANL 12FT and BNL 7FT restricted nuisance parameters, $f_{\mathrm{Re}}^{\mathrm{ANL}}(\nu)$ and $f_{\mathrm{Re}}^{\mathrm{BNL}}(\nu)$, have $\delta f=5 \% . \delta f^{\mathrm{ANL}}$ and $\delta f^{\mathrm{BNL}}$ are such that ANL 12FT and BNL 7FT nonreanalyzed datasets data are constrained by an overall $15 \%$ Gaussian. The full summary of the nuisance parameters is in Table V.

Priors are applied to the parameters of interest to penalize disagreement with well-established parameter values. For instance, the description of neutrino CC quasielastic cross sections and single-pion production through baryon resonances is strongly determined by the shape of the weakaxial and vector-transition form factors. As mentioned in Sec. IV B, the form factors are extracted from datasets not used in this analysis. Our goal is not the extraction of the axial masses but the better estimation of the cross section at the SIS region. For this reason, these values are used as priors in our global fits (see Table IV).

Another parameter of interest which is strongly constrained by data is the $S_{\text {DIS }}$ parameter. This parameter

TABLE V. Nuisance parameters, $f^{j}$, per experiment (ANL 12FT, BNL 7FT, BEBC, or FNAL 15FT) and neutrino beam $\left(\nu_{\mu}\right.$ or $\left.\bar{\nu}_{\mu}\right)$. Priors consider the systematic uncertainty applied to each dataset as $\delta f_{j}$, where $j$ is one of the datasets under study. The allowed range is $[0,2]$ for nuisance parameters considered in the tune. See the text for the details on the prior values.

\begin{tabular}{ll}
\hline \hline Parameter & Prior \\
\hline$f^{\mathrm{ANL}}\left(\nu_{\mu}\right)$ & $1 \pm 0.14$ \\
$f_{\mathrm{Re}}^{\mathrm{AN}}\left(\nu_{\mu}\right)$ & $1 \pm 0.05$ \\
$f^{\mathrm{BNL}}\left(\nu_{\mu}\right)$ & $1 \pm 0.14$ \\
$f_{\mathrm{Re}}^{\mathrm{BNL}}\left(\nu_{\mu}\right)$ & $1 \pm 0.05$ \\
$f^{\mathrm{BNL}}\left(\bar{\nu}_{\mu}\right)$ & $1 \pm 0.15$ \\
$f^{\mathrm{BEBC}}\left(\nu_{\mu}\right)$ & $1 \pm 0.15$ \\
$f^{\mathrm{BEBC}}\left(\bar{\nu}_{\mu}\right)$ & $1 \pm 0.15$ \\
$f^{\mathrm{FNAL}}\left(\nu_{\mu}\right)$ & $1 \pm 0.15$ \\
$f^{\mathrm{FNAL}}\left(\bar{\nu}_{\mu}\right)$ & $1 \pm 0.15$ \\
\hline \hline
\end{tabular}


dominates the cross-section behavior at high neutrino energies. Most of the data in that energy range comes from neutrino interactions with heavy nuclear targets and are therefore not included in the fit. A Gaussian prior is considered to ensure that agreement with these data are preserved [85] by our tuning procedure. This would not be the case otherwise as the SIS region data would prefer much higher cross-section values for the DIS contribution. The prior on $S_{\text {DIS }}$ provides a good solution for this problem because the degeneracy between DIS and nonresonance background parameters gives us multiple ways to accommodate good agreement between data and GENIE predictions in the SIS region. In other words, the introduction of the $S_{\text {DIS }}$ prior breaks the degeneracy without adding more datasets to the fit.

\section{Final form of the $\chi^{2}$}

Including all of the contributions from the previous sections and defining $\sigma_{d}^{i j k}\left(\delta \sigma_{\text {stat }}^{i j k}\right)$ as the data central value (statistical error) corresponding to the $\tilde{\sigma}_{\mathrm{th}}^{i j}\left(E_{k} \mid \boldsymbol{\theta}\right)$ prediction, the complete form of our $\chi^{2}$ distribution becomes

$$
\begin{aligned}
\chi^{2}(\boldsymbol{\theta}, \boldsymbol{f})= & \sum_{i, j, k} w^{i j k} \frac{\left(\phi_{j}(\boldsymbol{f}) \tilde{\sigma}_{\mathrm{th}}^{i j}\left(E_{k} \mid \boldsymbol{\theta}\right)-\sigma_{d}^{i j k}\right)^{2}}{\left(\delta \sigma_{\mathrm{stat}}^{i j k}\right)^{2}} \\
& +\left(\boldsymbol{\theta}-\boldsymbol{\theta}_{0}\right)^{T} \Sigma_{\theta}^{-1}\left(\boldsymbol{\theta}-\boldsymbol{\theta}_{0}\right) \\
& +\sum_{j} \frac{\left(f_{j}-1\right)^{2}}{\left(\delta f_{j}\right)^{2}}
\end{aligned}
$$

where $\phi_{j}(\boldsymbol{f})$ is the product of the nuisance scaling factors that are relevant for $j$ th dataset as described in Sec. V B. $\boldsymbol{\theta}_{0}$ and $\Sigma_{\theta}$ are the central values and the covariance matrix of the priors for the parameters of interest, respectively. Equation (10) represents the full capability of our tuning machinery. However, the priors we applied for the present work were uncorrelated and so only the diagonal entries of $\Sigma_{\theta}$ were used. The details on the priors applied in this analysis are described in Sec. V C.

The contribution of each point to the likelihood can be (de)emphasized using weights $w^{i j k}$ to set the relative importance of different datasets (or of individual data points within a dataset). Such weighting schemes have been used extensively in general-purpose event generator tunes for the LHC (for an example, see [86]). In this particular analysis, the weights are used to include or exclude datasets only ( $w^{i j k} \in\{0,1\}$ ).

\section{TUNING RESULTS}

In order to properly understand the global tune, the tensions between datasets must be discussed. The identification of these tensions were instrumental to motivate a global tuning procedure and the usage of priors as described in previous sections to respect the agreement with all the datasets used in the fit at the same time. These tensions are studied by performing fits using a specific dataset to evaluate the impact of the partially-fitted predictions on the rest of the datasets included in the global tune.

In the following sections, different fit results are compared against a number of datasets, including those not used in the fit. Each dataset is identified by a label and Table XI provides the proper references for each one of them. Some of those data are not extracted from (anti) neutrino interactions on hydrogen or deuterium, yet they are included for historical reasons. This explains why some datasets are not compatible among themselves and reinforces the need for free nucleon tune only. Table XI reports the target composition used for each dataset.

\section{A. Partial fits}

Two main subsets were identified in the global dataset in order to study tensions; inclusive and exclusive datasets. The fits consider the G18_02a CMC as the base configuration and include nuisance parameters to take into account the correlation between datasets from the same experiment (see Sec. V for more details). No priors on $M_{A}^{\mathrm{RES}}$ and $M_{A}^{\mathrm{QE}}$ are applied as we are interested to see the impact of each subset on the prediction. The fit to inclusive data only is not sensitive to the scaling multiplicity parameters for the nonresonant background, therefore those parameters are fixed to their default values during the fit.

Partial fit results for inclusive and exclusive data are presented in Table VI. The tune against inclusive data only achieves much better agreement with inclusive data than GENIE G18_02a_00_000 (see Fig. 6). This difference between the old and new inclusive tune is due to (1) the inclusion of only hydrogen and deuterium datasets, and (2) the effect of the nuisance parameters [87] Particularly, without exclusive data, a small reduction of the resonant cross section is already observed in the CC RES region. The result for $M_{A}^{\mathrm{RES}}$ is consistent with previous results

TABLE VI. Parameter best-fit results for partial fits to inclusive and exclusive data using the G18_02a CMC as base configuration. Values within parentheses are kept fixed during the fit; they are the historical default values.

\begin{tabular}{lcc}
\hline \hline Parameter & Inclusive & Exclusive \\
\hline$W_{\text {cut }}(\mathrm{GeV})$ & 1.52 & 2.00 \\
$M_{A}^{\mathrm{QE}}\left(\mathrm{GeV} / \mathrm{c}^{2}\right)$ & $0.98 \pm 0.01$ & $1.003 \pm 0.008$ \\
$M_{A}^{\mathrm{RES}}\left(\mathrm{GeV} / \mathrm{c}^{2}\right)$ & $1.15 \pm 0.02$ & $0.88 \pm 0.02$ \\
$R_{\nu p}^{\mathrm{CC} 1 \pi}$ & $(0.10)$ & $0.30 \pm 0.02$ \\
$R_{\nu p}^{\mathrm{CC} 2 \pi}$ & $(1.00)$ & $1.28 \pm 0.06$ \\
$R_{\nu n}^{\mathrm{CC} 1 \pi}$ & $(0.30)$ & $0.294 \pm 0.002$ \\
$R_{\nu n}^{\mathrm{CC} 2 \pi}$ & $(1.00)$ & $3.19 \pm 0.09$ \\
$S_{\mathrm{RES}}$ & $0.87 \pm 0.03$ & $0.88 \pm 0.02$ \\
$S_{\mathrm{DIS}}$ & $1.027 \pm 0.005$ & $1.026 \pm 0.007$ \\
\hline \hline
\end{tabular}




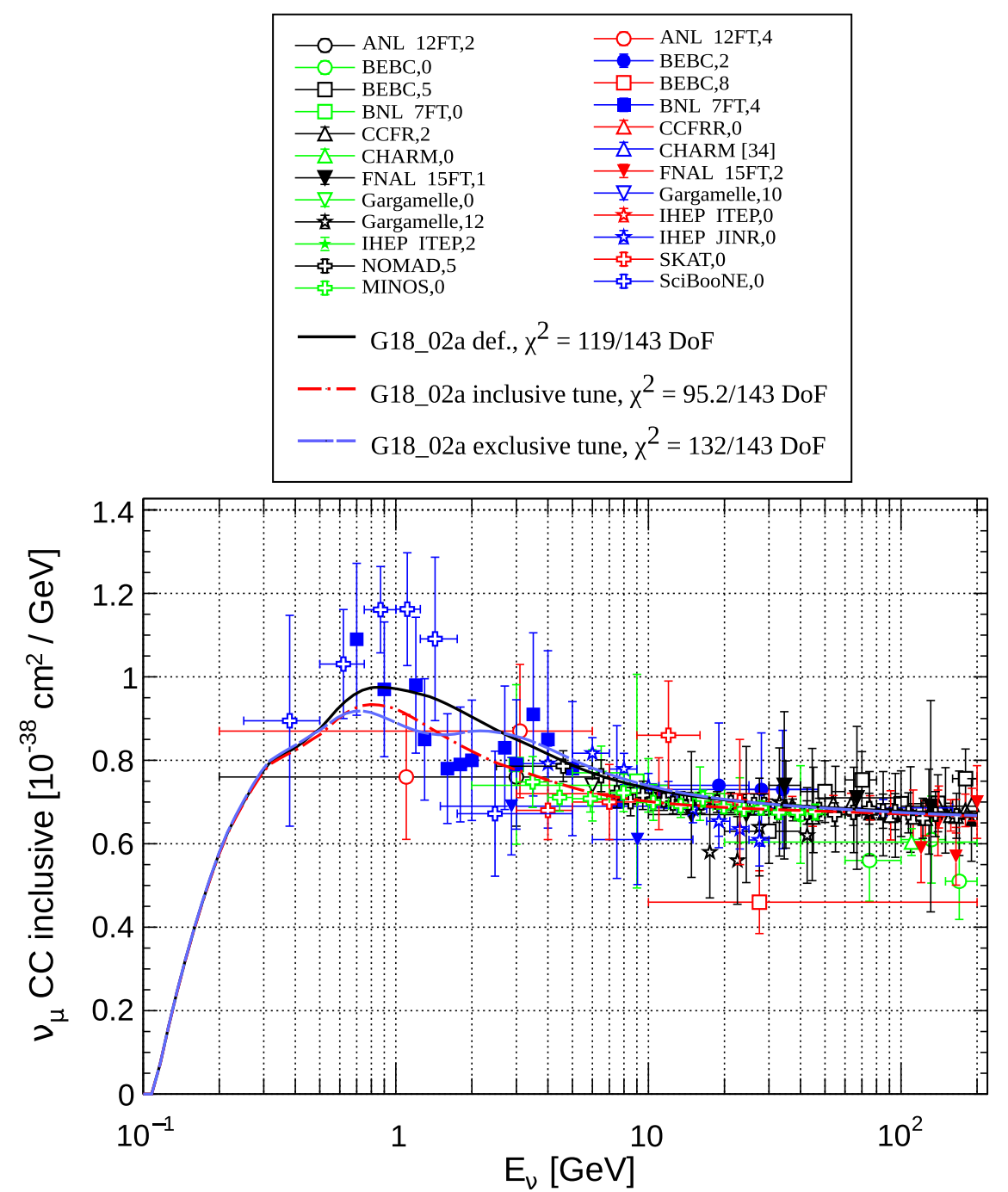

FIG. 6. Comparison of $\nu_{\mu} \mathrm{CC}$ inclusive cross section against bubble chamber data. The default GENIE configuration corresponds to the G18_02a_00_000 CMC. The inclusive tune is performed using the filled data points only. The predictions are computed with GENIE version 3.2 using the parameters specified in Table VI. The $\chi^{2}$ values are calculated against all inclusive data available from bubble chamber experiments.

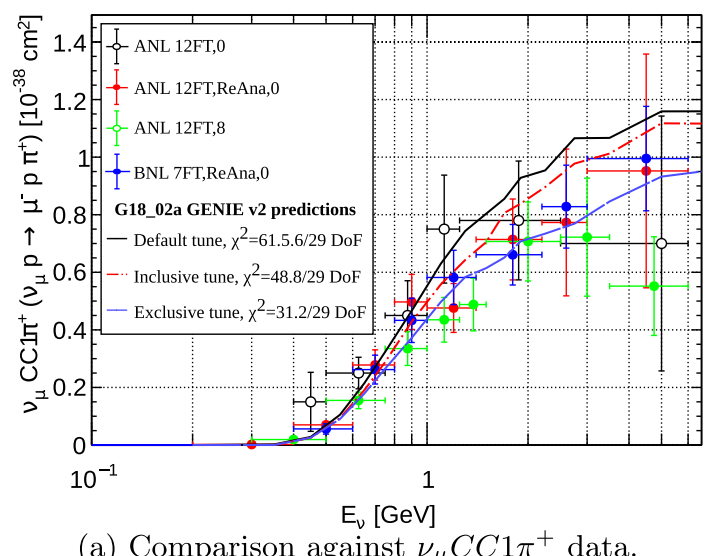

(a) Comparison against $\nu_{\mu} C C 1 \pi^{+}$data.

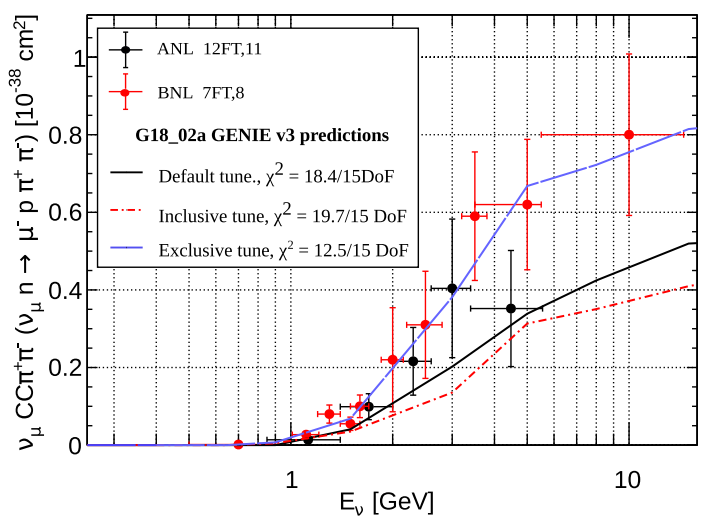

(b) Comparison against $\nu_{\mu} C C \pi^{+} \pi^{-}$data.

FIG. 7. Comparison of $\nu_{\mu}$ CC exclusive channels against bubble chamber data. The default GENIE configuration corresponds to the G18_02a_00_000 CMC. The exclusive tune is sensitive to the exclusive datasets only (see Table III). The predictions are computed with GENIE version 3.2 using the parameters specified in Table VI. The $\chi^{2}$ values are calculated against all exclusive data shown in each plot. 
TABLE VII. Best-fit parameter values and parameter ranges obtained by requiring that $\Delta \chi_{\text {profiled }}^{2}<\Delta \chi_{\text {critical }}^{2}=1$. Results are shown for all alternative $\mathrm{CMCs}$ considered in this analysis. The bestfit values obtained for the G18_02a(/b) CMC can be used for the $\mathrm{G} 18 \_10 \mathrm{a}(/ \mathrm{b})$ as the same bare-nucleon underlying models are used.

\begin{tabular}{lcc}
\hline \hline Parameter & G18_01a(/b) & G18_02a(/b) \\
\hline$W_{\text {cut }}$ & 1.94 & 1.81 \\
$M_{A}^{\mathrm{QE}}$ & $1.00 \pm 0.01$ & $1.00 \pm 0.013$ \\
$M_{A}^{\mathrm{RES}}$ & $1.09 \pm 0.02$ & $1.09 \pm 0.014$ \\
$R_{\nu p}^{\mathrm{CC} 1 \pi}$ & $0.06 \pm 0.03$ & 0.008 \\
$R_{\nu p}^{\mathrm{CC} 2 \pi}$ & $1.1 \pm 0.2$ & $0.94 \pm 0.075$ \\
$R_{\nu n}^{\mathrm{CC} 1 \pi}$ & $0.14 \pm 0.03$ & $0.03 \pm 0.010$ \\
$R_{\nu n}^{\mathrm{CC} 2 \pi}$ & $2.8 \pm 0.4$ & $2.3 \pm 0.12$ \\
$S_{\mathrm{RES}}$ & $0.89 \pm 0.04$ & $0.84 \pm 0.028$ \\
$S_{\mathrm{DIS}}$ & $1.03 \pm 0.02$ & $1.06 \pm 0.01$ \\
$\chi^{2} / 157 \mathrm{DoF}$ & 1.84 & 1.64 \\
\hline \hline
\end{tabular}

TABLE VIII. Best-fit nuisance parameters, $f^{j}$, per experiment (ANL 12FT, BNL 7FT, BEBC or FNAL 15FT) and neutrino beam $\left(\nu_{\mu}\right.$ or $\left.\bar{\nu}_{\mu}\right)$. The nuisance parameters included in the fit are independent of GENIE.

\begin{tabular}{llr}
\hline \hline Parameter & G18_01a(/b) & G18_02a(/b) \\
\hline$f^{\mathrm{ANL}}\left(\nu_{\mu}\right)$ & $0.98 \pm 0.05$ & $0.89 \pm 0.05$ \\
$f_{\mathrm{Re}}^{\mathrm{ANL}}\left(\nu_{\mu}\right)$ & $1.12 \pm 0.05$ & $1.2 \pm 0.05$ \\
$f^{\mathrm{BNL}}\left(\nu_{\mu}\right)$ & $1.01 \pm 0.04$ & $1.06 \pm 0.04$ \\
$f_{\mathrm{Re}}^{\mathrm{BNL}}\left(\nu_{\mu}\right)$ & $1.08 \pm 0.05$ & $1.03 \pm 0.04$ \\
$f^{\mathrm{BNL}}\left(\bar{\nu}_{\mu}\right)$ & $1.00 \pm 0.10$ & $0.99 \pm 0.10$ \\
$f^{\mathrm{BEBC}}\left(\nu_{\mu}\right)$ & $0.91 \pm 0.04$ & $0.86 \pm 0.03$ \\
$f^{\mathrm{BEBC}}\left(\bar{\nu}_{\mu}\right)$ & $1.04 \pm 0.04$ & $0.99 \pm 0.03$ \\
$f^{\mathrm{FNAL}}\left(\nu_{\mu}\right)$ & $0.97 \pm 0.04$ & $0.94 \pm 0.04$ \\
$f^{\mathrm{FNAL}}\left(\bar{\nu}_{\mu}\right)$ & $0.95 \pm 0.05$ & $0.92 \pm 0.05$ \\
\hline \hline
\end{tabular}

\begin{tabular}{|c|c|}
\hline 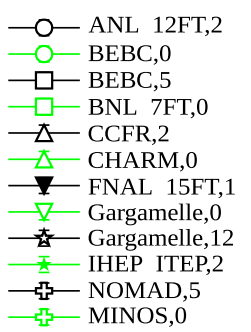 & 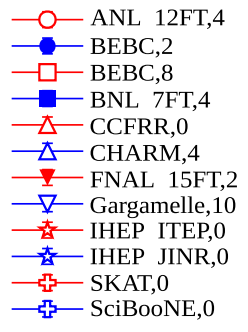 \\
\hline \multicolumn{2}{|c|}{$\longrightarrow$ G18_02a def., $x^{2}=119 / 143$ DoF } \\
\hline \multicolumn{2}{|c|}{ G18_01a tuned, $x^{2}=99.7 / 143$ DoF } \\
\hline — G18_02a tu & 123/143 DoF \\
\hline
\end{tabular}

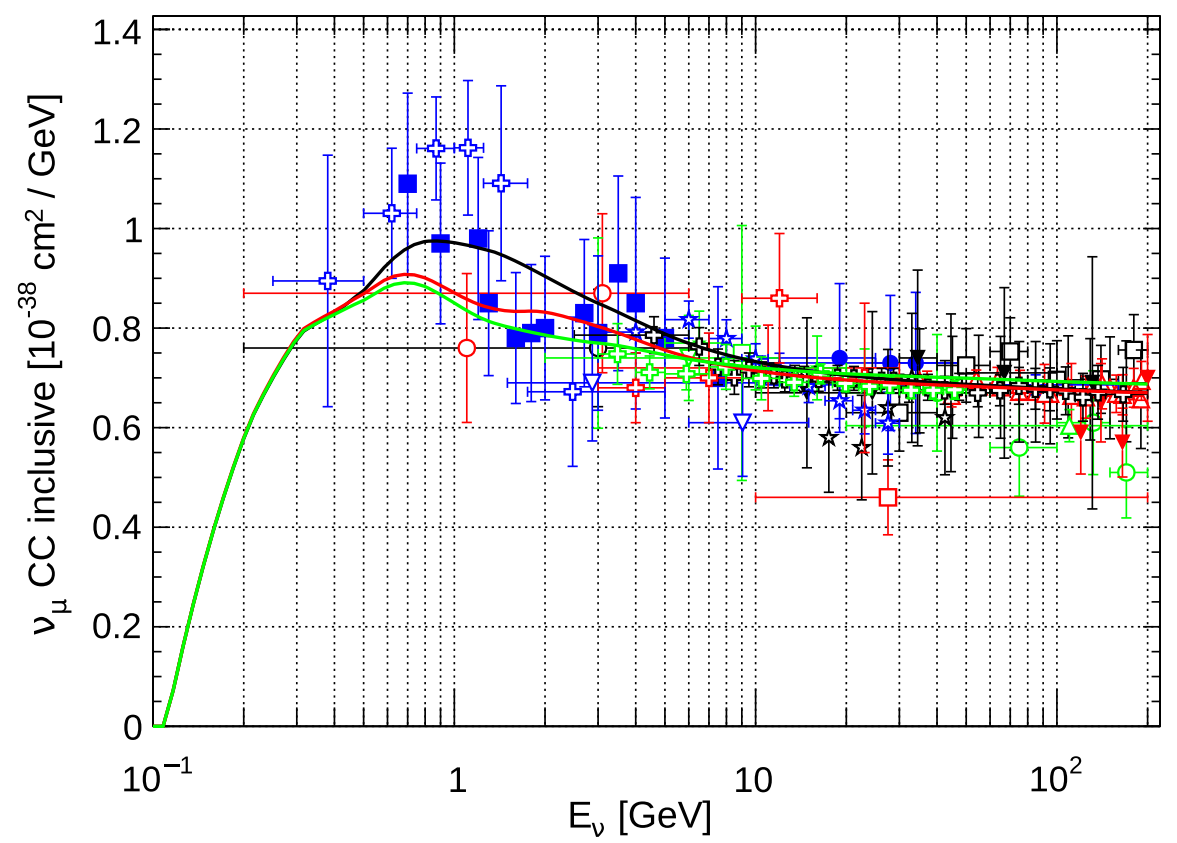

FIG. 8. Best-fit prediction impact on muon neutrino CC inclusive cross sections as a function of the neutrino energy $\left(E_{\nu}\right)$. The associated predictions for the default G18_02a and tuned G18_01a and G18_02a are computed with GENIE v3.0.6. Predictions are compared against all the available data (anti)neutrino interactions on $\mathrm{H},{ }^{2} \mathrm{H}$, and heavier targets. Both CMCs have been tuned against some $\mathrm{H},{ }^{2} \mathrm{H}$ data (filled markers). Each $\chi^{2}$ is computed using all data available. In Table $\mathrm{X}$, the $\chi^{2}$ values per dataset are specified. 
without the addition of priors [80]. $W_{\text {cut }}$ is pulled to the lower edge of the parameter range; the parameter uncertainty could not be estimated as the $\chi^{2}$ minimum was found on the contour.

As expected, the fit to exclusive data only is able to correctly describe exclusive datasets for one- and two-pion production. The low cross-section data for one-pion production forces all the relevant parameters to decrease with respect to the default values [see Fig. 7(a)]. At the same time, two pion production data forces $R_{\nu p}^{\mathrm{CC} 2 \pi}, R_{\nu n}^{\mathrm{CC} 2 \pi}$ and $W_{\text {cut }}$ to increase in order to match two pion production data [see Fig. 7(b)]. The agreement with $\nu_{\mu} \mathrm{CC}$ inclusive data is worse, see Fig. 6, but the compatibility is still acceptable given the large uncertainties on the data in that region. On the other hand, the partial fit does not obtain a good prediction for $M_{A}^{\mathrm{RES}}$. $W_{\text {cut }}$ is fixed to its maximum value of $2 \mathrm{GeV}$ to avoid nonphysical regions.

The exclusive fit clearly shows a preference for a larger total cross section in the neutrino energy region between 1 and $10 \mathrm{GeV}$ due to the high value of $R_{\nu p}^{\mathrm{CC} 2 \pi}$ and $R_{\nu n}^{\mathrm{CC} 2 \pi}$. This is a tension between exclusive and inclusive datasets as the inclusive prefer a lower value in that $E_{\nu}$ region. Since inclusive data constitute about $40 \%$ of all the data points, the inclusion of priors for the axial masses and $S_{\text {DIS }}$ becomes crucial to overcome the tension [88].

\section{B. Global fit}

The analysis procedure outlined in previous sections was applied to the comprehensive model configurations listed in

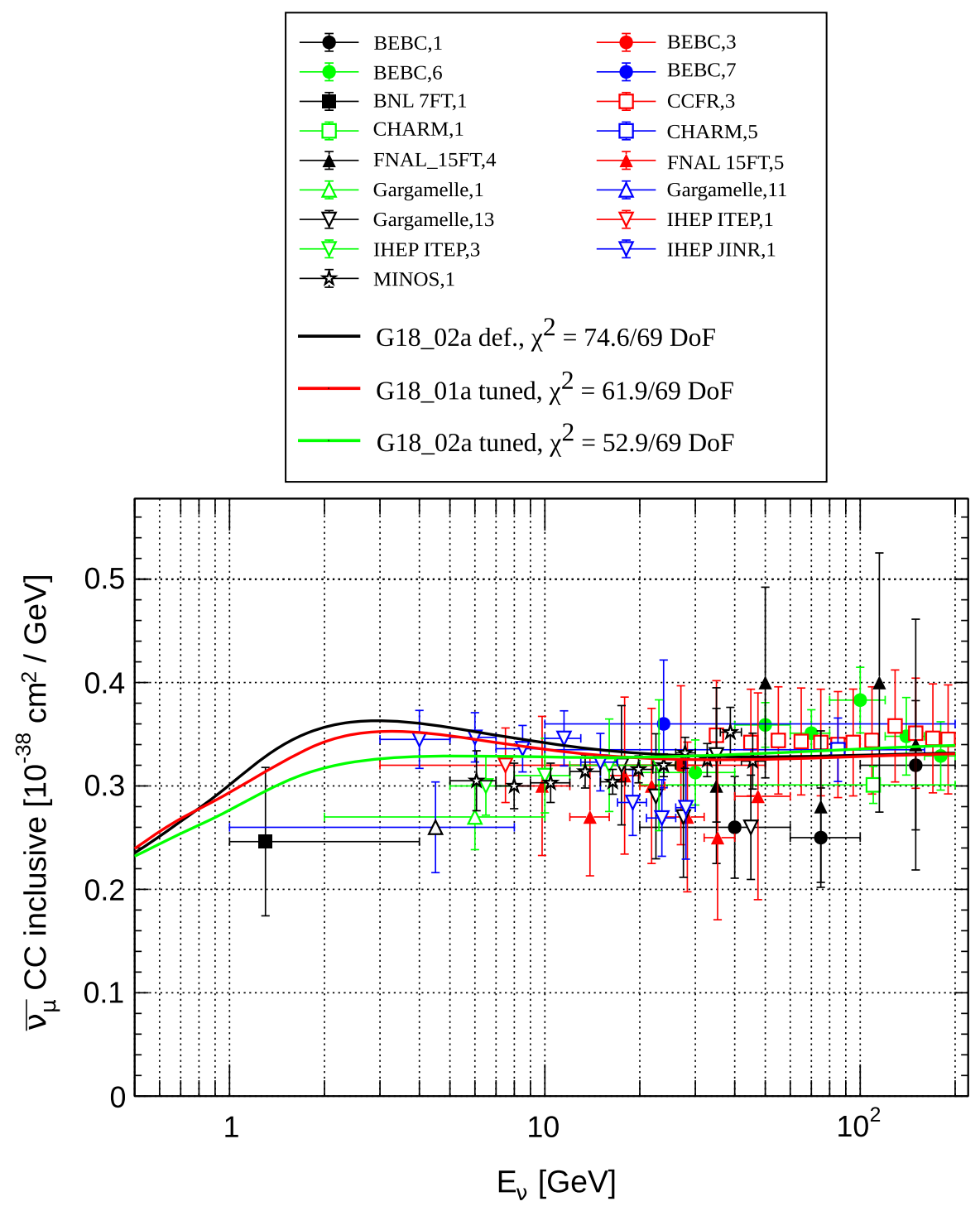

FIG. 9. Best-fit prediction impact on muon antineutrino $\mathrm{CC}$ inclusive cross sections as a function of the neutrino energy ( $\left.E_{\nu}\right)$. The associated predictions for the default G18_02a and tuned G18_01a and G18_02a are computed with GENIE v3.0.6. Predictions are compared against all the available data (anti)neutrino interactions on $\mathrm{H},{ }^{2} \mathrm{H}$, and heavier targets. Both CMCs have been tuned against some $\mathrm{H},{ }^{2} \mathrm{H}$ data (filled markers). Each $\chi^{2}$ is computed using all data available. In Table $\mathrm{X}$, the $\chi^{2}$ values per dataset are specified. 
Sec. III. The best-fit parameter values obtained from the GENIE analysis for each alternative CMC are shown in Tables VII and VIII. The GENIE v3 cross-section curves that correspond to the two sets of tuned parameters are shown in Figs. 8-13. For reference, we also show the crosssection predictions made by the G18_02a_00_000 CMC that are available in the last public release of the GENIE v3 series (3.2).

For all CMCs the tune has the most impact on the SIS region. In the inclusive cross-section prediction, this translates into a decrease of both $\nu_{\mu}$ and $\bar{\nu}_{\mu} \mathrm{CC}$ inclusive cross section in the $0.5-10 \mathrm{GeV}$ region (see Figs. 8 and 9). At the same time, the cross section at higher neutrino energies has barely changed, respecting the constraints of high-energy data. The agreement with quasielastic data, included in the tune in order to constrain the fluxes of each experiment, remained the same (see Fig. 10).

As discussed in Sec. VI, this decrease of the inclusive cross section at the SIS region is driven mainly by one-pion production data. The impact on one-pion exclusive channels is shown for (anti)neutrino on proton, Fig. 11, and neutrino on neutron, Fig. 12. The reduction of the one-pion production cross section for neutrino on proton and neutron shows an improvement on $\nu_{\mu} \mathrm{CC} 1 \pi^{+}, \nu_{\mu}$ and $\nu_{\mu} \mathrm{CC} 1 \pi^{-}$and $\nu_{\mu} \mathrm{CC} 1 \pi^{0}$ channels when comparing it with the available data.

Two-pion production exclusive cross sections are summarized in Fig. 13. This is the first time that two-pion production data are used to tune the SIS region, allowing the $R_{\nu p}^{\mathrm{CC} 2 \pi}$ and $R_{\nu n}^{\mathrm{CC} 2 \pi}$ parameters to be constrained. In this case, the two-pion exclusive cross section was

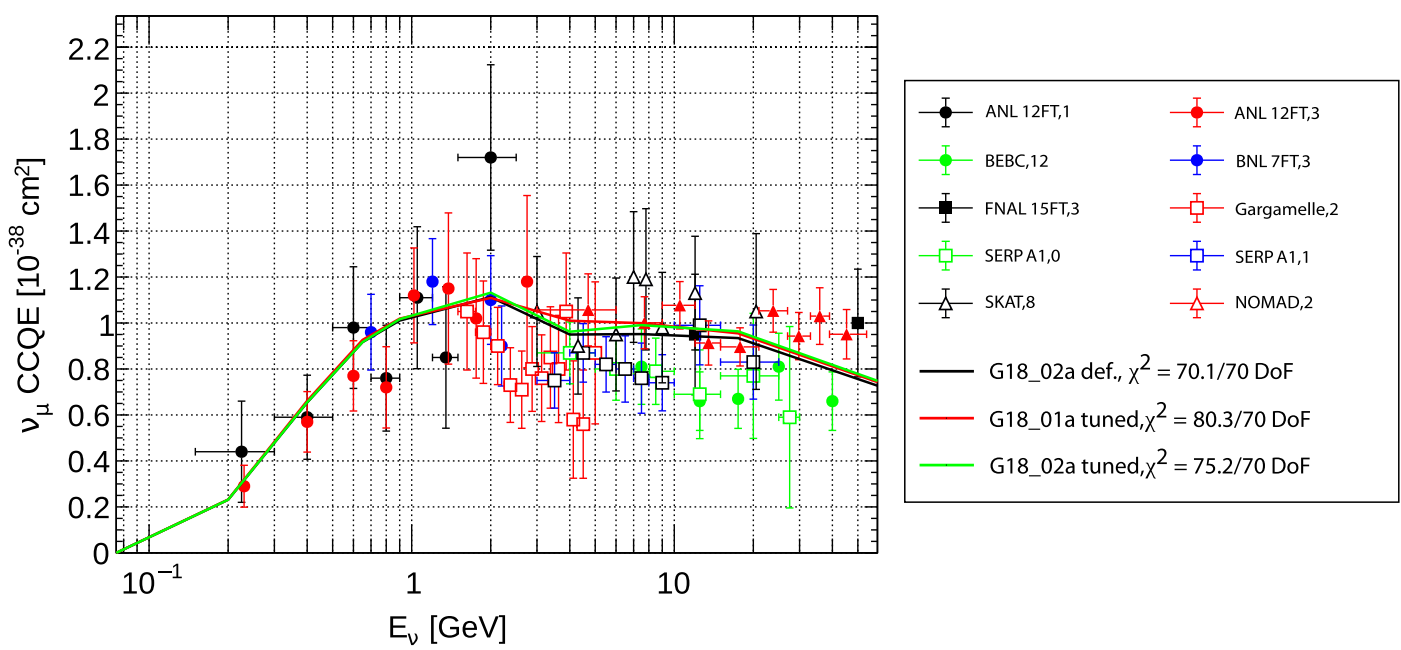

(a) Comparison of $\nu_{\mu}$ CC quasi-elastic cross-section data against the default and tuned CMCs.

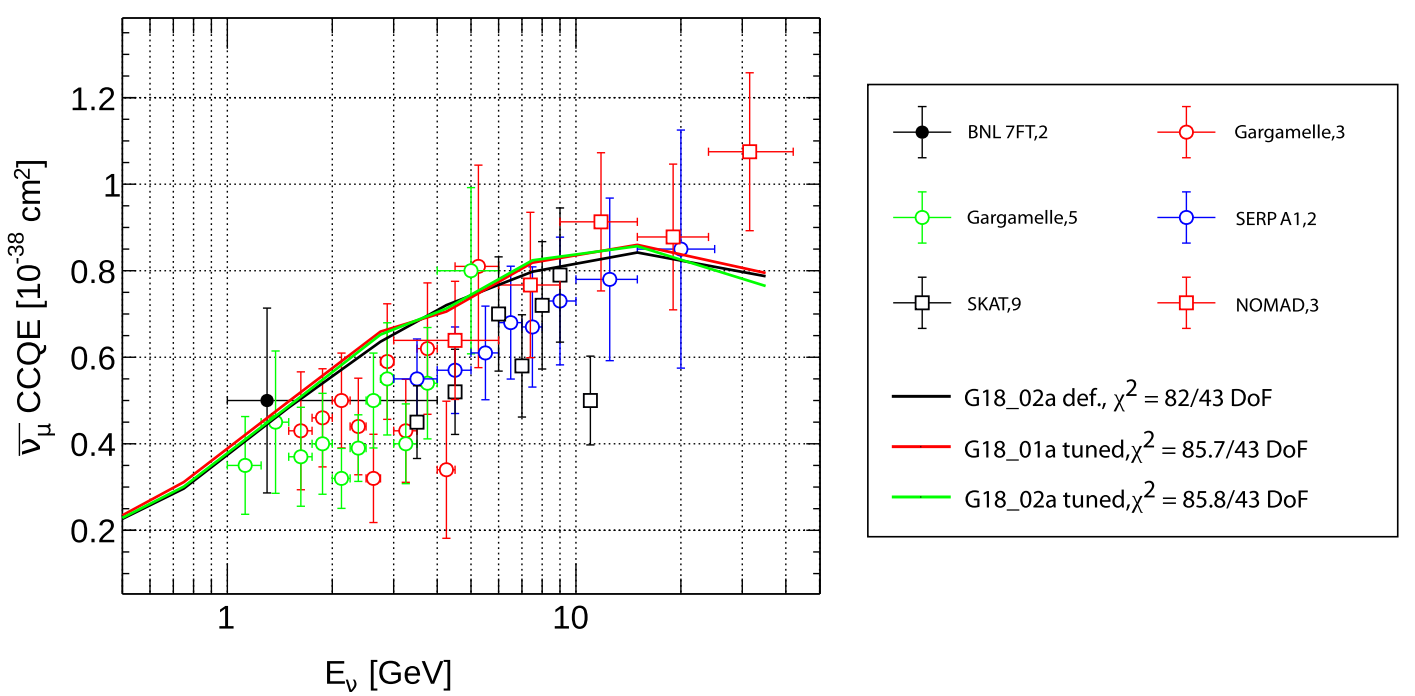

(b) Comparison of $\bar{\nu}_{\mu}$ CC quasi-elastic cross-section data against the default and tuned CMCs.

FIG. 10. Best-fit prediction impact on muon (anti)neutrino CC quasielastic cross sections as a function of the neutrino energy $\left(E_{\nu}\right)$. The associated predictions for the default G18_02a, and tuned G18_01a and G18_02a are computed with GENIE v3.0.6. Predictions are compared against all the available data (anti)neutrino interactions on $\mathrm{H},{ }^{2} \mathrm{H}$, and heavier targets. Both $\mathrm{CMCs}$ have been tuned against some $\mathrm{H},{ }^{2} \mathrm{H}$ data (filled markers). Each $\chi^{2}$ is computed using all data available. In Table $\mathrm{X}$, the $\chi^{2}$ values per dataset are specified. 


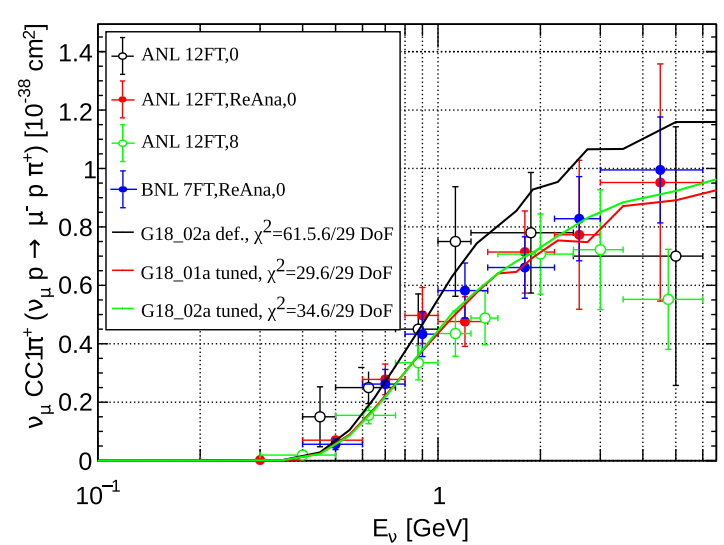

(a) Comparison of $\nu_{\mu} \mathrm{CC} 1 \pi^{+}$data on proton against the default and tuned CMCs.

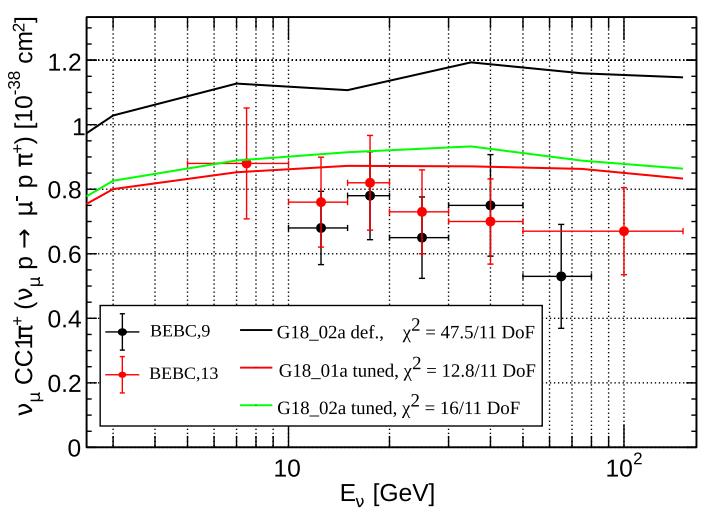

(c) Comparison of $\nu_{\mu} \mathrm{CC} 1 \pi^{+}$data on proton against the default and tuned CMCs.

Experimental analysis impose a cut on $W$ at $2 \mathrm{GeV}$.

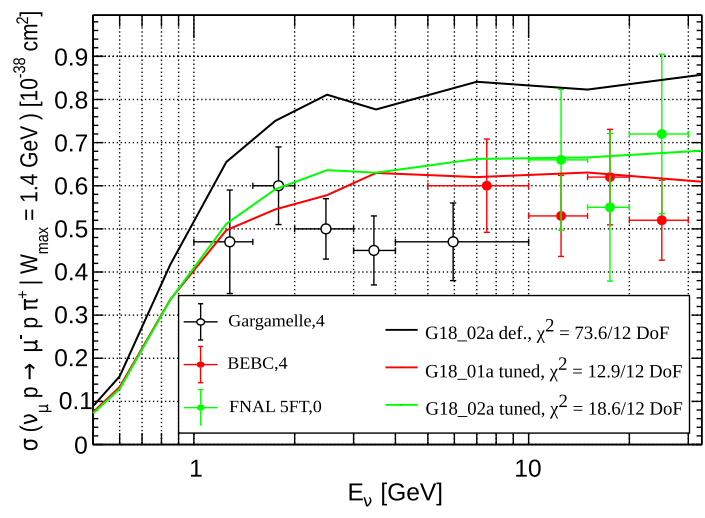

(b) Comparison of $\nu_{\mu} \mathrm{CC} 1 \pi^{+}$data on proton against the default and tuned CMCs.

Experimental analysis impose a cut on $W$ at $1.4 \mathrm{GeV}$.

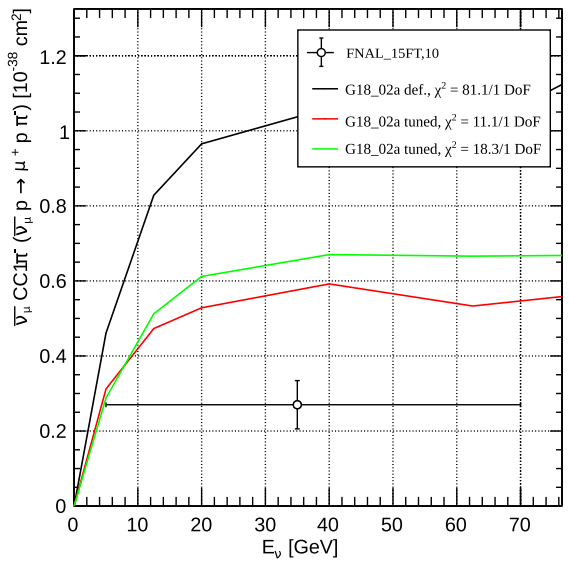

(d) Comparison of $\bar{\nu}_{\mu}$ CC $1 \pi^{+}$data on proton against the default and tuned CMCs. The FNAL 15FT experiment applied a cut on $W$ at $1.9 \mathrm{GeV}$

FIG. 11. Best-fit prediction impact on muon neutrino on proton CC one-pion production cross sections as a function of the neutrino energy $\left(E_{\nu}\right)$. The associated predictions for the default G18_02a and tuned G18_01a and G18_02a are computed with GENIE v3.0.6. Experimental cuts are also applied to the predictions when needed. Predictions are compared against all the available data (anti)neutrino interactions on $\mathrm{H},{ }^{2} \mathrm{H}$, and heavier targets. Both CMCs have been tuned against some $\mathrm{H},{ }^{2} \mathrm{H}$ data (filled markers). Each $\chi^{2}$ is computed using all data available. In Table $\mathrm{X}$, the $\chi^{2}$ values per dataset are specified.

underestimated by the default tune. For this particular exclusive process, comparisons are made against $\nu_{\mu} \mathrm{CC} \pi^{+} \pi^{+}, \nu_{\mu} \mathrm{CC} \pi^{+} \pi^{0}$, and $\nu_{\mu} \mathrm{CC} \pi^{+} \pi^{-}$data. The shape of the GENIE prediction for the $\nu_{\mu} \mathrm{CC} \pi^{+} \pi^{+}$and $\nu_{\mu} \mathrm{CC} \pi^{+} \pi^{0}$ channels differs strongly from the data, and the models are not able to accommodate this behavior. However, the agreement with $\nu_{\mu} \mathrm{CC} \pi^{+} \pi^{-}$data has improved by increasing the cross section with respect to the default $\mathrm{CMC}$.

Despite the tensions between inclusive and exclusive data discussed in Sec. IV B, the overall agreement for both cross-section model constructions has improved (see Table X). Particularly, $\bar{\nu}_{\mu} \mathrm{CC}$ inclusive predictions show better agreement after the tune, and the same is observed for $\nu_{\mu} \mathrm{CC}$ inclusive predictions for the G18_01a free nucleon tune. Although the impact on the cross-section prediction of the tune is similar for the existing configurations, the response of each model at the parameter level is not expected to be the same. Therefore, each tune is strongly affected by how the model is able to accommodate the data by modifying the tuned parameters. This reflects on the $R_{m}$ parameters and $W_{\text {cut }}$ which best-fit values are incompatible in some cases, such as for $R_{\nu n}^{\mathrm{CC} 1 \pi}$ (see Table VII). Particularly, the behavior of $R_{\nu p}^{\mathrm{CC} 1 \pi}$ on the G18_02a(/b) tune was showing preference for nonphysical regions of the tune, forcing us to fix this value to $R_{\nu p}^{\mathrm{CC} 1 \pi}=0.008$. On the other hand, the remaining parameters, such as $M_{A}^{\mathrm{RES}}$ and $M_{A}^{\mathrm{QE}}$, show agreement between the tunes and respect the applied priors. 


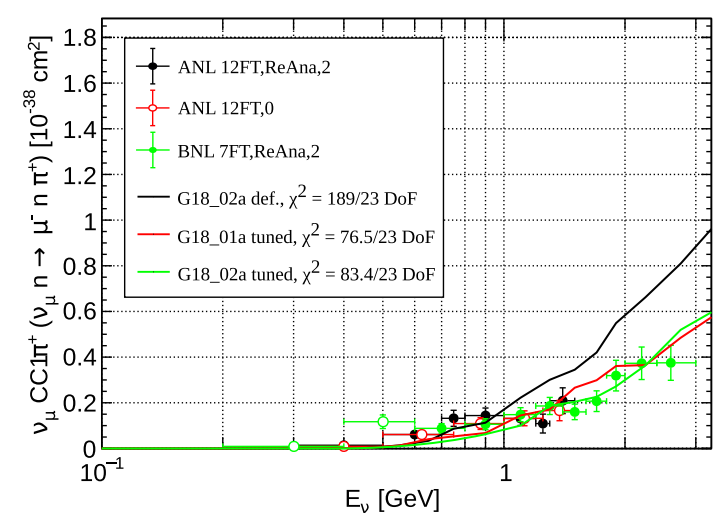

(a) Comparison of $\nu_{\mu}$ CC $1 \pi^{+}$data on neutron against the default and tuned CMCs.

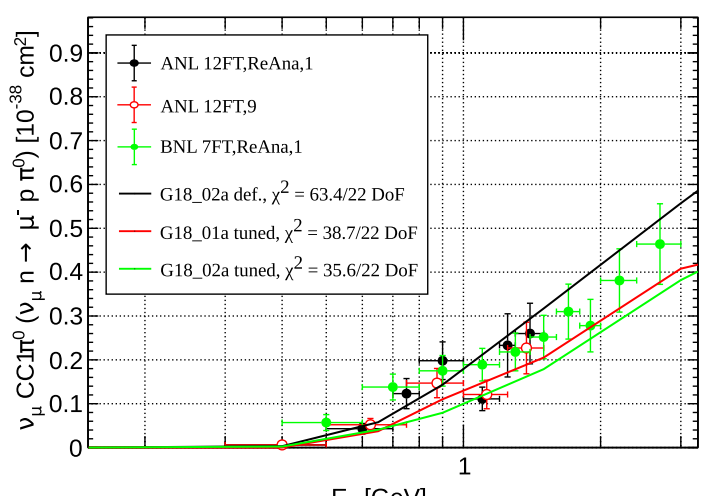

$\mathrm{E}_{\mathrm{v}}[\mathrm{GeV}]$

(b) Comparison of $\nu_{\mu} \mathrm{CC} 1 \pi^{0}$ data on neutron against the default and tuned CMCs.

FIG. 12. Best-fit prediction impact on muon neutrino on neutron CC one-pion production cross sections as a function of the neutrino energy $\left(E_{\nu}\right)$. The associated predictions for the default G18_02a and tuned G18_01a and G18_02a are computed with GENIE v3.0.6. Predictions are compared against the original and reanalyzed ANL 12FT and BNL 7FT data [68,69]. Only reanalyzed data with $E_{\nu}>0.5 \mathrm{GeV}$ is used in the tune (filled markers). Each $\chi^{2}$ is computed using all data available.

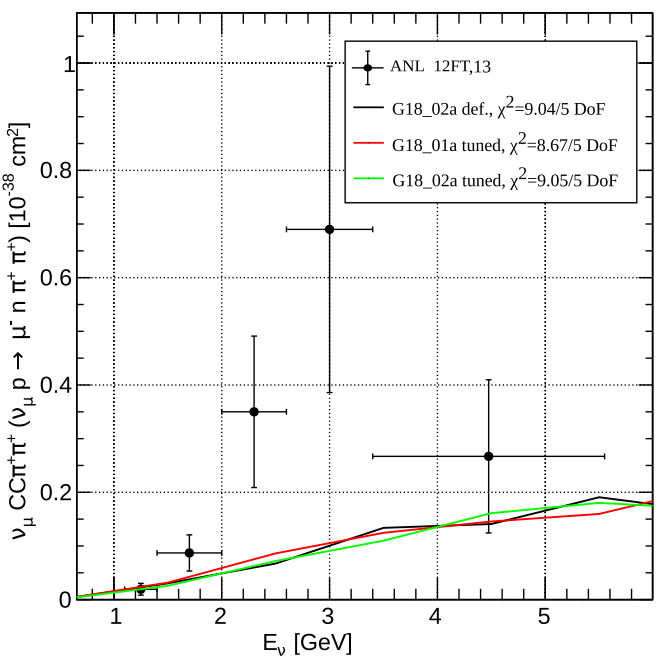

(a) Comparison of $\nu_{\mu}$ CC $2 \pi^{+}$data on proton.

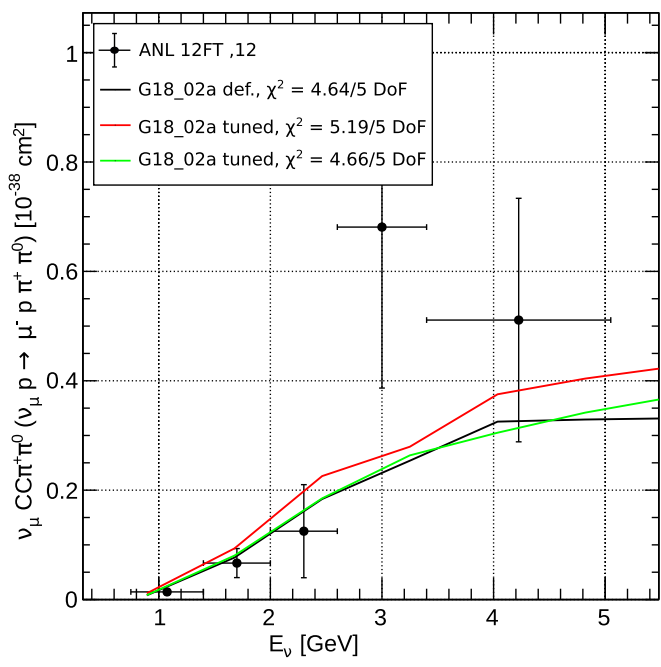

(b) Comparison of $\nu_{\mu} \mathrm{CC} \pi^{+} \pi^{0}$ data on proton.

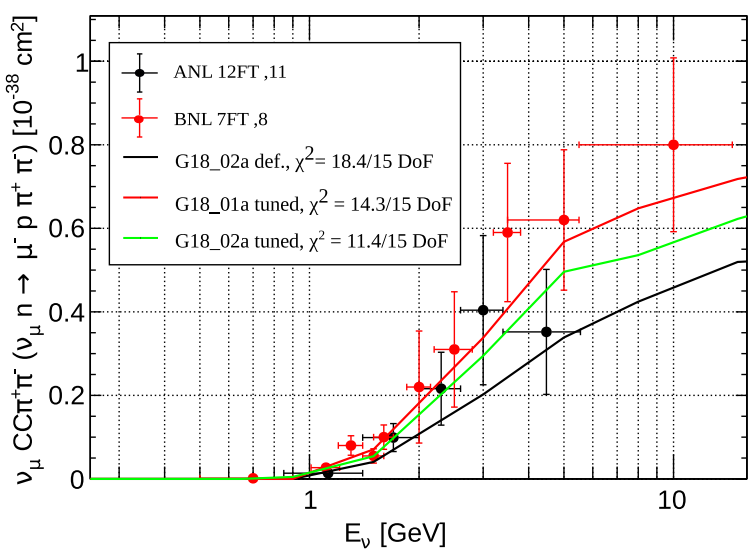

(c) Comparison of $\nu_{\mu} \mathrm{CC} \pi^{+} \pi^{-}$data on neutron.

FIG. 13. Best-fit prediction impact on muon neutrino CC two-pion production cross sections as a function of the neutrino energy $\left(E_{\nu}\right)$. The comparisons to two-pion production data are shown against the default and tuned CMCs. The associated predictions for the default G18_02a and tuned G18_01a and G18_02a are computed with GENIE v3.0.6. Predictions are compared against ANL 12FT and BNL 7FT data. 


\section{Parameter error estimation}

An estimate of the parameter uncertainties is shown in Table VII. For each parameter of interest allowed to float in the fit, the table shows the range of values that satisfies the condition $\Delta \chi_{\text {profile }}^{2}\left(\theta_{i}\right)<\Delta \chi_{\text {critical }}^{2}=1$. In the previous expression, the function $\Delta \chi_{\text {profile }}^{2}\left(\theta_{i}\right)$ is constructed by fixing $\theta_{i}$ to a desired value and minimizing the quantity
$\Delta \chi^{2}(\boldsymbol{\theta}, \boldsymbol{f})=\chi^{2}(\boldsymbol{\theta}, \boldsymbol{f})-\chi_{\min }^{2}$ with respect to all other parameters that were allowed to float in the fit. [See Sec. VA for the definition of $\left.\chi^{2}(\boldsymbol{\theta}, \boldsymbol{f})\right]$. The constant $\chi_{\min }^{2}$ corresponds to the minimum value of $\chi^{2}(\boldsymbol{\theta}, \boldsymbol{f})$ obtained from the global fit. The $\Delta \chi_{\text {profile }}^{2}\left(\theta_{i}\right)$ functions we derive from our analysis are shown in Fig. 14, for all parameters $\theta_{i}$ that were allowed to float in the fit, up to $\Delta \chi_{\text {profile }}^{2}$ values of 2. Particularly,

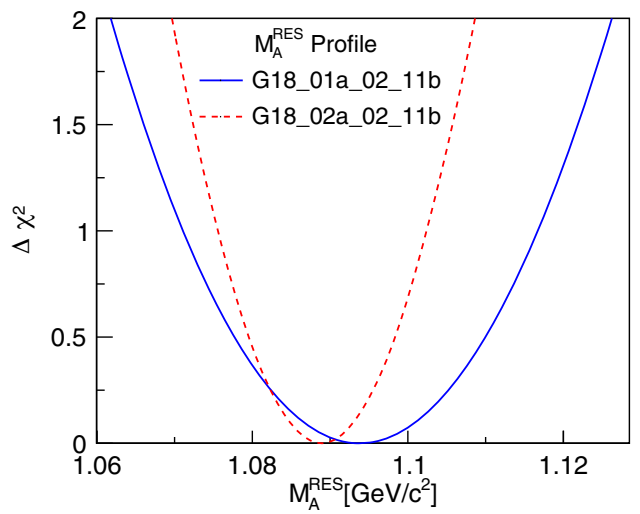

(b) Profile for $M_{A}^{\mathrm{RES}}$.

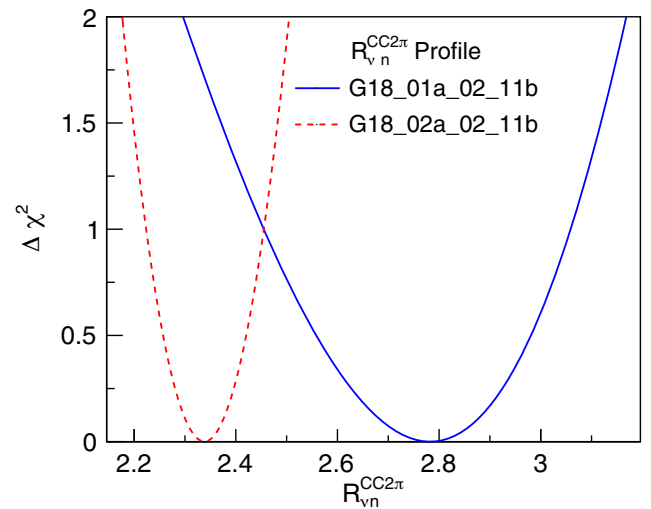

(d) Profile for $R_{\nu n}^{\mathrm{CC} 2 \pi}$.

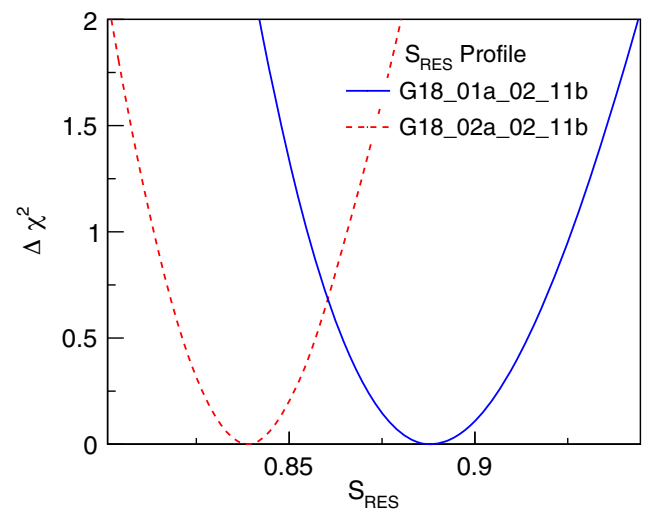

(f) Profile for $S_{\text {RES }}$.

FIG. 14. $\Delta \chi_{\text {profile }}^{2}\left(\theta_{i}\right)$ functions obtained fixing the parameter under study and minimizing $\Delta \chi^{2}\left(\theta_{i}\right)=\chi^{2}(\vec{\theta}, \vec{f})-\chi_{\min }^{2}$ respect the other parameters in the tune. The profiles for both tunes are shown for each case. The G18_02a profiles show that this configuration is less able to accommodate in the model variations on each parameter best-fit values. 
TABLE IX. Parameter covariance matrices extracted the GENIE fit for the tuned CMCs.

\begin{tabular}{|c|c|c|c|c|c|c|c|c|c|}
\hline \multicolumn{10}{|c|}{ G18_01a(/b) covariance matrix } \\
\hline & $M_{A}^{\mathrm{RES}}$ & $M_{A}^{\mathrm{QE}}$ & $R_{\nu p}^{\mathrm{CC} 1 \pi}$ & \multicolumn{2}{|c|}{$R_{\nu p}^{\mathrm{CC} 2 \pi}$} & $R_{\nu n}^{\mathrm{CC} 1 \pi}$ & $R_{\nu n}^{\mathrm{CC} 2 \pi}$ & $S_{\mathrm{RES}}$ & $S_{\text {DIS }}$ \\
\hline$M_{A}^{\mathrm{RES}}$ & $5.3 \times 10^{-4}$ & $-7 \times 10^{-5}$ & & \multicolumn{2}{|c|}{$-8 \times 10^{-4}$} & $2.2 \times 10^{-4}$ & $-2.4 \times 10^{-3}$ & $-4.3 \times 10^{-4}$ & $-9 \times 10^{-5}$ \\
\hline$M_{4}^{\mathrm{QE}}$ & $-7 \times 10^{-5}$ & $1.2 \times 10^{-4}$ & $-6 \mathrm{E} \times 10^{-5}$ & \multicolumn{2}{|c|}{$-1.2 \times 10^{-4}$} & $-5 \times 10^{-5}$ & $-7.6 \times 10^{-4}$ & $1.2 \times 10^{-4}$ & $1 \times 10^{-5}$ \\
\hline$R_{\nu p}^{\mathrm{CC} 1 \pi}$ & $5 \times 10^{-5}$ & $-6 \times 10^{-5}$ & $9.3 \times 10^{-4}$ & \multicolumn{2}{|c|}{$-1.6 \times 10^{-3}$} & $2.6 \times 10^{-4}$ & $5.4 \times 10^{-4}$ & $-2.8 \times 10^{-4}$ & $-6 \times 10^{-5}$ \\
\hline$R_{\nu p}^{\mathrm{CC} 2 \pi}$ & $-8 \times 10^{-4}$ & $-1.2 \times 10^{-4}$ & $-1.6 \times 10^{-3}$ & \multicolumn{2}{|c|}{$2.7 \times 10^{-2}$} & $2.0 \times 10^{-5}$ & $-2.5 \times 10^{-4}$ & $2 \times 10^{-3}$ & $-6.2 \times 10^{-4}$ \\
\hline$R_{\nu n}^{\mathrm{CC} 1 \pi}$ & $2.2 \times 10^{-4}$ & $-5 \times 10^{-5}$ & $2.6 \times 10^{-4}$ & \multicolumn{2}{|c|}{$2 \times 10^{-5}$} & $7.1 \times 10^{-4}$ & $2.3 \times 10^{-3}$ & $-5.3 \times 10^{-4}$ & $-8 \times 10^{-5}$ \\
\hline$R_{\nu n}^{\mathrm{CC} 2 \pi}$ & $-2.4 \times 10^{-3}$ & $-7.6 \times 10^{-4}$ & $5.4 \times 10^{-4}$ & \multicolumn{2}{|c|}{$-2.5 \times 10^{-4}$} & $2.3 \times 10^{-3}$ & $9.6 \times 10^{-2}$ & $-2.5 \times 10^{-3}$ & $-1.4 \times 10^{-3}$ \\
\hline$S_{\mathrm{RES}}$ & $-4.3 \times 10^{-4}$ & $1.2 \times 10^{-4}$ & $-2.8 \times 10^{-4}$ & \multicolumn{2}{|c|}{$2 \times 10^{-3}$} & $-5.3 \times 10^{-4}$ & $-2.5 \times 10^{-3}$ & $1.3 \times 10^{-3}$ & $1.8 \times 10^{-4}$ \\
\hline$S_{\text {DIS }}$ & $-9 \times 10^{-5}$ & $1 \times 10^{-5}$ & $-6 \times 10^{-5}$ & \multicolumn{2}{|c|}{$-6.2 \times 10^{-4}$} & $-8 \times 10^{-5}$ & $-1.4 \times 10^{-3}$ & $1.8 \times 10^{-4}$ & $5.1 \times 10^{-4}$ \\
\hline \multicolumn{10}{|c|}{ G18_02a(/b) covariance matrix } \\
\hline & $M_{A}^{\mathrm{RES}}$ & $M_{A}^{\mathrm{QE}}$ & & & \multicolumn{2}{|c|}{$R_{\nu n}^{\mathrm{CC} 1 \pi}$} & $R_{\nu n}^{\mathrm{CC} 2 \pi}$ & $S_{\text {RES }}$ & $S_{\text {DIS }}$ \\
\hline$M_{A}^{\mathrm{RES}}$ & $1.7 \times 10^{-4}$ & $2.0 \times 10^{-5}$ & \multicolumn{2}{|c|}{$-1.9 \times 10^{-4}$} & \multicolumn{2}{|c|}{$-6.0 \times 10^{-5}$} & $4.4 \times 10^{-4}$ & $6.0 \times 10^{-5}$ & $-4.0 \times 10^{-5}$ \\
\hline$M_{A}^{\mathrm{QE}}$ & $2.0 \times 10^{-5}$ & $1.8 \times 10^{-4}$ & $-7.0 \times$ & $10^{-5}$ & 3.0 & $\times 10^{-5}$ & $-2.1 \times 10^{-4}$ & $1.5 \times 10^{-4}$ & $1.0 \times 10^{-5}$ \\
\hline$R_{\nu p}^{\mathrm{CC} 2 \pi}$ & $-1.9 \times 10^{-4}$ & $-7.0 \times 10^{-5}$ & $5.5 \times$ & $10^{-3}$ & 1.5 & $\times 10^{-4}$ & $-2.4 \times 10^{-3}$ & $-6.9 \times 10^{-4}$ & $3.0 \times 10^{-5}$ \\
\hline$R_{\nu n}^{\mathrm{CC} 1 \pi}$ & $-6.0 \times 10^{-5}$ & $3.0 \times 10^{-5}$ & $1.5 \times$ & $10^{-4}$ & & $\times 10^{-4}$ & $-1.0 \times 10^{-4}$ & $-6.0 \mathrm{E}-5$ & $6.0 \times 10^{-5}$ \\
\hline$R_{\nu n}^{\mathrm{CC} 2 \pi}$ & $4.4 \times 10^{-4}$ & $-2.1 \times 10^{-4}$ & $-2.4 \times$ & $10^{-3}$ & -1.0 & $\times 10^{-4}$ & $1.3 \times 10^{-2}$ & $2.0 \times 10^{-4}$ & $-8.0 \times 10^{-5}$ \\
\hline$S_{\mathrm{RES}}$ & $6.0 \times 10^{-5}$ & $1.5 \times 10^{-4}$ & $-6.9 \times$ & $10^{-4}$ & -6.0 & $\times 10^{-5}$ & $2.3 \times 10^{-4}$ & $6.0 \times 10^{-4}$ & $-4.0 \times 10^{-5}$ \\
\hline$S_{\text {DIS }}$ & $-4.0 \times 10^{-5}$ & $1.0 \times 10^{-5}$ & $3.0 \times$ & $10^{-5}$ & 6.0 & $\times 10^{-5}$ & $-8.0 \times 10^{-5}$ & $-4.0 \times 10^{-5}$ & $8.0 \times 10^{-5}$ \\
\hline
\end{tabular}

TABLE X. Contributions to the default and tuned $\chi^{2}$ for the datasets included in the fit. The data points with $E_{\nu}<0.5 \mathrm{GeV}$, a total of 10 points, are considered in the $\chi^{2}$ calculations of this table, but were not used in the fit. For the calculation of the $\chi^{2}$, the covariance matrix between the datasets is used instead of Eq. (10), which incorporates nuisance parameters which are not implemented in GENIE. This explains the difference when comparing with the $\chi^{2}$ out of Professor from Table VII.

\begin{tabular}{|c|c|c|c|c|c|}
\hline \multirow[b]{2}{*}{ Dataset } & \multirow[b]{2}{*}{$N_{\text {DOF }}$} & \multicolumn{2}{|c|}{$\chi^{2}$ values for G18_01a } & \multicolumn{2}{|c|}{$\chi^{2}$ values for G18_02a } \\
\hline & & Default & Best fit & Default & Best fit \\
\hline \multicolumn{6}{|l|}{$\nu_{\mu} \mathrm{CC}$ Inclusive } \\
\hline BNL 7FT [45] & 13 & 11.1 & 9.95 & 14.7 & 7.75 \\
\hline BEBC [43] & 3 & 0.215 & 0.101 & 0.067 & 0.045 \\
\hline FNAL 15FT $[48,75]$ & 10 & 3.85 & 3.92 & 4.04 & 4.98 \\
\hline \multicolumn{6}{|l|}{$\bar{\nu}_{\mu} \mathrm{CC}$ Inclusive } \\
\hline BEBC $[32,43,44]$ & 11 & 11.17 & 11.5 & 9.79 & 9.8 \\
\hline BNL 7FT [54] & 1 & 1.83 & 1.51 & 1.96 & 0.827 \\
\hline FNAL 15FT [50,55] & 13 & 3.86 & 4.12 & 4.32 & 4.13 \\
\hline \multicolumn{6}{|l|}{$\nu_{\mu} n \rightarrow \mu^{-} n \pi^{+}$} \\
\hline ANL 12FT [68] & 5 & 11.6 & 9.88 & 27.3 & 14.3 \\
\hline ANL 12FT,ReAna [69] & 7 & 31.3 & 21.0 & 48.8 & 25.2 \\
\hline BNL 7FT,ReAna [69] & 11 & 103 & 45.7 & 112 & 43.9 \\
\hline \multicolumn{6}{|l|}{$\nu_{\mu} p \rightarrow \mu^{-} p \pi^{+}$} \\
\hline ANL 12FT,ReAna [69] & 8 & 11 & 8.71 & 17.8 & 9.64 \\
\hline BNL 7FT,ReAna [69] & 7 & 6.16 & 3.11 & 9.71 & 3.9 \\
\hline BEBC $[59,71,73]$ & 15 & 33.98 & 15.9 & 82.6 & 21.0 \\
\hline FNAL [78] & 3 & 1.11 & 0.74 & 2.87 & 0.66 \\
\hline \multicolumn{6}{|l|}{$\nu_{\mu} n \rightarrow \mu^{-} p \pi^{0}$} \\
\hline ANL 12FT [68] & 5 & 4.89 & 4.98 & 7.57 & 4.63 \\
\hline ANL 12FT,ReAna [69] & 7 & 12.6 & 12.0 & 17.4 & 11.5 \\
\hline BNL 7FT,ReAna [69] & 10 & 31.8 & 21.7 & 38.4 & 19.4 \\
\hline
\end{tabular}


TABLE X. (Continued)

\begin{tabular}{|c|c|c|c|c|c|}
\hline \multirow[b]{2}{*}{ Dataset } & \multirow[b]{2}{*}{$N_{\text {DOF }}$} & \multicolumn{2}{|c|}{$\chi^{2}$ values for G18_01a } & \multicolumn{2}{|c|}{$\chi^{2}$ values for G18_02a } \\
\hline & & Default & Best fit & Default & Best fit \\
\hline \multicolumn{6}{|l|}{$\nu_{\mu} p \rightarrow \mu^{-} n \pi^{+} \pi^{+}$} \\
\hline ANL 12FT [76] & 5 & 9.23 & 8.67 & 9.04 & 9.05 \\
\hline \multicolumn{6}{|l|}{$\nu_{\mu} p \rightarrow \mu^{-} p \pi^{+} \pi^{0}$} \\
\hline ANL 12FT [76] & 5 & 4.28 & 5.19 & 4.64 & 4.66 \\
\hline \multicolumn{6}{|l|}{$\nu_{\mu} n \rightarrow \mu^{-} p \pi^{+} \pi^{-}$} \\
\hline ANL 12FT [76] & 5 & 8.24 & 8.36 & 8.09 & 4.95 \\
\hline BNL 7FT [36] & 10 & 11.6 & 5.96 & 10.3 & 6.46 \\
\hline \multicolumn{6}{|l|}{$\nu_{\mu} \mathrm{CC} \mathrm{QE}$} \\
\hline ANL 12FT [30,58] & 15 & 11.7 & 12.2 & 11.75 & 11.58 \\
\hline BNL 7FT [63] & 4 & 6.88 & 6.91 & 6.98 & 7.58 \\
\hline BEBC [59] & 5 & 8.18 & 9.45 & 8.21 & 9.54 \\
\hline FNAL [60] & 2 & 0.886 & 0.951 & 0.992 & 0.893 \\
\hline \multicolumn{6}{|l|}{$\bar{\nu}_{\mu} \mathrm{CC} \mathrm{QE}$} \\
\hline BNL 7FT [54] & 1 & 0.161 & 0.135 & 0.078 & 0.106 \\
\hline Total & 182 & 400.6 & 229.5 & 459.4 & 236.5 \\
\hline
\end{tabular}

TABLE XI. Summary of data used for comparisons in Figs. 6, 7(a), 7(b), 8, 9, 10, 11, 13, 12, and 17. This table links the experiment and the tag used for the legend in each plot to the corresponding reference. It also provides the composition of the active volume of the experiment.

\begin{tabular}{lccr}
\hline \hline Experiment & Target & Tag & References \\
\hline & & & \\
ANL 12 FT & ${ }^{1} \mathrm{H},{ }^{2} \mathrm{H}$ & $\nu_{\mu}$ CC Inclusive & {$[30]$} \\
BEBC & ${ }^{2} \mathrm{H}, \mathrm{Ne}$ & ANL 12 FT, 2 & {$[31]$} \\
BEBC & ${ }^{2} \mathrm{H}, \mathrm{Ne}$ & BEBC,0 & {$[32]$} \\
BNL 7FT & ${ }^{2} \mathrm{H}, \mathrm{Ne}$ & BEBC,5 & {$[33]$} \\
CCFR & $\mathrm{Fe}$ & BNL 7FT, 0 & {$[34]$} \\
CHARM & $\mathrm{C}$ & CCFR,2 & {$[35]$} \\
FNAL 15FT & ${ }^{2} \mathrm{H}$ & CHARM,0 & {$[36]$} \\
Gargamelle & $\mathrm{C}_{3} \mathrm{H}_{8}, \mathrm{CF}_{3} \mathrm{Br}$ & Gargamelle, 0 & {$[37]$} \\
Gargamelle & $\mathrm{C}_{3} \mathrm{H}_{8}, \mathrm{CF}_{3} \mathrm{Br}$ & Gargamelle,12 & {$[38]$} \\
IHEP_ITEP & $\mathrm{Fe}$ & IHEP_ITEP,2 & {$[39]$} \\
NOMAD & $\mathrm{C}$ & NOMAD,5 & {$[40]$} \\
MINOS & $\mathrm{C}$ & MINOS,0 & {$[41]$} \\
ANL 12FT & $\mathrm{C}$ & ANL 12FT,4 & {$[42]$} \\
BEBC & ${ }^{1} \mathrm{H},{ }^{2} \mathrm{H}$ & BEBC,2 & {$[43]$} \\
BEBC & ${ }^{1} \mathrm{H}, \mathrm{Ne}$ & BEBC,8 & {$[44]$} \\
BNL 7FT & ${ }^{1} \mathrm{H}, \mathrm{Ne}$ & BNL 7FT,4 & {$[45]$} \\
CCFRR & ${ }^{2} \mathrm{H}$ & CCFRR,0 & {$[46]$} \\
CHARM & $\mathrm{Fe}$ & CHARM,4 & {$[47]$} \\
FNAL 15FT & $\mathrm{C}$ & FNAL 15FT,2 & {$[48]$} \\
Gargamelle & ${ }^{1} \mathrm{H}, \mathrm{Ne}$ & Gargamelle,10 & {$[49]$} \\
IHEP_ITEP & $\mathrm{C}_{3} \mathrm{H}_{8}, \mathrm{CF}{ }_{3} \mathrm{Br}$ & IHEP_ITEP,0 & {$[39]$} \\
IHEP_JINR & $\mathrm{Fe}$ & IHEP_JINR,0 & {$[51]$} \\
SKAT & $\mathrm{Al}$ & SKAT,0 & {$[52]$} \\
SciBooNE & $\mathrm{CF}_{3} \mathrm{Br}$ & SciBooNE,0 & {$[53]$} \\
\hline & $\mathrm{C}$ & & (Table continued)
\end{tabular}


TABLE XI. (Continued)

\begin{tabular}{|c|c|c|c|}
\hline Experiment & Target & Tag & References \\
\hline \multicolumn{4}{|c|}{$\bar{\nu}_{\mu}$ CC Inclusive } \\
\hline BEBC & ${ }^{1} \mathrm{H}, \mathrm{Ne}$ & BEBC,1 & [31] \\
\hline BEBC & ${ }^{1} \mathrm{H}, \mathrm{Ne}$ & BEBC,6 & [32] \\
\hline BNL 7FT & ${ }^{1} \mathrm{H}$ & BNL 7FT,1 & {$[54]$} \\
\hline CHARM & $\mathrm{C}$ & CHARM,1 & {$[35]$} \\
\hline FNAL 15FT & ${ }^{2} \mathrm{H}, \mathrm{Ne}$ & FNAL 15FT,4 & [55] \\
\hline Gargamelle & $\mathrm{C}_{3} \mathrm{H}_{8}, \mathrm{CF}_{3} \mathrm{Br}$ & Gargamelle,1 & {$[37]$} \\
\hline Gargamelle & $\mathrm{C}_{3} \mathrm{H}_{8}, \mathrm{CF}_{3} \mathrm{Br}$ & Gargamelle, 13 & [38] \\
\hline IHEP_ITEP & $\mathrm{Fe}$ & IHEP_ITEP,3 & [39] \\
\hline MINOS & $\mathrm{C}$ & MIN̄OS,1 & [41] \\
\hline BEBC & ${ }^{1} \mathrm{H}, \mathrm{Ne}$ & $\mathrm{BEBC}, 3$ & [43] \\
\hline BEBC & ${ }^{1} \mathrm{H}, \mathrm{Ne}$ & BEBC,7 & [44] \\
\hline CCFR & $\mathrm{Fe}$ & CCFR,3 & [34] \\
\hline CHARM & $\mathrm{C}$ & CHARM,5 & {$[47]$} \\
\hline FNAL 15FT & ${ }^{2} \mathrm{H}, \mathrm{Ne}$ & FNAL 15FT,5 & {$[50]$} \\
\hline Gargamelle & $\mathrm{C}_{3} \mathrm{H}_{8}, \mathrm{CF}_{3} \mathrm{Br}$ & Gargamelle,11 & {$[56]$} \\
\hline IHEP_ITEP & $\mathrm{Fe}$ & IHEP_ITEP,1 & [57] \\
\hline IHEP_JINR & $\mathrm{Fe}$ & IHEP_JINR,1 & {$[51]$} \\
\hline Experiment & Target & Tag & References \\
\hline \multicolumn{4}{|c|}{$\nu_{\mu} \mathrm{CC}$ Quasielastic } \\
\hline ANL 12FT & ${ }^{2} \mathrm{H}$ & ANL 12FT,1 & {$[58]$} \\
\hline $\mathrm{BEBC}$ & ${ }^{2} \mathrm{H}$ & $\mathrm{BEBC}, 12$ & [59] \\
\hline FNAL 15FT & ${ }^{2} \mathrm{H}$ & FNAL 15FT,3 & {$[60]$} \\
\hline SERP A1 & $\mathrm{Al}$ & SERP A1,0 & [61] \\
\hline SKAT & $\mathrm{CF}_{3} \mathrm{Br}$ & SKAT,8 & {$[62]$} \\
\hline ANL 12FT & ${ }^{1} \mathrm{H},{ }^{2} \mathrm{H}$ & ANL 12FT,3 & {$[30]$} \\
\hline BNL 7FT & ${ }^{2} \mathrm{H}$ & BNL 7FT,3 & [63] \\
\hline Gargamelle & $\mathrm{C}_{3} \mathrm{H}_{8}, \mathrm{CF}_{3} \mathrm{Br}$ & Gargamelle,2 & [64] \\
\hline SERP A1 & $\mathrm{Al}$ & SERP A1,1 & {$[65]$} \\
\hline NOMAD & $\mathrm{C}$ & NOMAD,2 & [40] \\
\hline \multicolumn{4}{|c|}{$\bar{\nu}_{\mu}$ CC Quasielastic } \\
\hline BNL 7FT & ${ }^{1} \mathrm{H}$ & BNL 7FT,2 & [54] \\
\hline Gargamelle & $\mathrm{C}_{3} \mathrm{H}_{8}, \mathrm{CF}_{3} \mathrm{Br}$ & Gargamelle,5 & {$[66]$} \\
\hline SKAT & $\mathrm{C}_{3} \mathrm{H}_{8}$ & SKAT,9 & {$[62]$} \\
\hline Gargamelle & $\mathrm{CF}_{3} \mathrm{Br}$ & Gargamelle,3 & {$[64]$} \\
\hline SERP A1 & $\mathrm{Al}$ & SERP A1,2 & {$[65]$} \\
\hline NOMAD & $\mathrm{C}$ & NOMAD,3 & [40] \\
\hline \multicolumn{4}{|c|}{$\nu_{\mu} \mathrm{CC} 1 \pi^{+}\left(\nu_{\mu} p \rightarrow \mu^{-} p \pi^{+}\right)$} \\
\hline ANL 12FT & ${ }^{2} \mathrm{H}$ & ANL 12FT,0 & {$[68]$} \\
\hline ANL 12FT & ${ }^{2} \mathrm{H}$ & ANL 12FT,ReAna,0 & [69] \\
\hline ANL 12FT & ${ }^{2} \mathrm{H}$ & ANL 12FT,8 & [69] \\
\hline BNL 7FT & ${ }^{2} \mathrm{H}$ & BNL 7FT,ReAna,0 & [69] \\
\hline Gargamelle & $\mathrm{C}_{3} \mathrm{H}_{8}, \mathrm{CF}_{3} \mathrm{Br}$ & Gargamelle, 4 & {$[70]$} \\
\hline $\mathrm{BEBC}$ & ${ }^{1} \mathrm{H}$ & $\mathrm{BEBC}, 4$ & {$[71]$} \\
\hline FNAL 15FT & ${ }^{1} \mathrm{H}$ & FNAL 15FT,0 & [72] \\
\hline $\mathrm{BEBC}$ & ${ }^{1} \mathrm{H}$ & BEBC,9 & [73] \\
\hline BEBC & ${ }^{2} \mathrm{H}$ & BEBC, 13 & [59] \\
\hline \multicolumn{4}{|c|}{$\bar{\nu}_{\mu} \mathrm{CC} 1 \pi^{-}\left(\bar{\nu} p \rightarrow \mu^{+} p \pi^{-}\right)$} \\
\hline FNAL 15FT & ${ }^{1} \mathrm{H}$ & FNAL 15FT,10 & {$[74]$} \\
\hline
\end{tabular}


TABLE XI. (Continued)

\begin{tabular}{|c|c|c|c|}
\hline Experiment & Target & Tag & References \\
\hline \multicolumn{4}{|c|}{$\nu_{\mu} \mathrm{CC} 1 \pi^{+}\left(\nu n \rightarrow \mu^{-} n \pi^{+}\right)$} \\
\hline ANL $12 \mathrm{FT}$ & ${ }^{1} \mathrm{H},{ }^{2} \mathrm{H}$ & ANL $12 \mathrm{FT}, \operatorname{ReAna}, 2$ & [69] \\
\hline BNL 7FT & ${ }^{2} \mathrm{H}$ & BNL 7FT,ReAna,2 & [69] \\
\hline ANL 12FT & ${ }^{1} \mathrm{H},{ }^{2} \mathrm{H}$ & ANL 12FT,10 & [68] \\
\hline \multicolumn{4}{|c|}{$\nu_{\mu} \mathrm{CC} 1 \pi^{0}\left(\nu n \rightarrow \mu^{-} p \pi^{0}\right)$} \\
\hline ANL $12 \mathrm{FT}$ & ${ }^{1} \mathrm{H},{ }^{2} \mathrm{H}$ & ANL 12FT,ReAna, 1 & [69] \\
\hline BNL 7FT & ${ }^{2} \mathrm{H}$ & BNL 7FT,ReAna,1 & [69] \\
\hline ANL $12 \mathrm{FT}$ & ${ }^{1} \mathrm{H},{ }^{2} \mathrm{H}$ & ANL $12 \mathrm{FT}, 9$ & [68] \\
\hline \multicolumn{4}{|c|}{$\nu_{\mu} \mathrm{CC} 1 \pi^{+} \pi^{+}\left(\nu p \rightarrow \mu^{-} n \pi^{+} \pi^{+}\right)$} \\
\hline ANL $12 \mathrm{FT}$ & ${ }^{2} \mathrm{H}$ & ANL 12FT,13 & [76] \\
\hline \multicolumn{4}{|c|}{$\nu_{\mu} \mathrm{CC} 1 \pi^{+} \pi^{0}\left(\nu p \rightarrow \mu^{-} p \pi^{+} \pi^{0}\right)$} \\
\hline ANL $12 \mathrm{FT}$ & ${ }^{2} \mathrm{H}$ & ANL 12FT,12 & [76] \\
\hline \multicolumn{4}{|c|}{$\nu_{\mu} \mathrm{CC} 1 \pi^{+} \pi^{-}\left(\nu n \rightarrow \mu^{-} p \pi^{+} \pi^{-}\right)$} \\
\hline ANL $12 \mathrm{FT}$ & ${ }^{2} \mathrm{H}$ & ANL 12FT,12 & [76] \\
\hline BNL 7FT & ${ }^{2} \mathrm{H}$ & BNL 7FT,8 & [75] \\
\hline
\end{tabular}

$W_{\text {cut }}$ is fixed to the best-fit value during this approach, as it is an ad hoc parameter introduced by the generator; by fixing it, its uncertainty will be reflected on the other parameters. It is important to emphasize that the uncertainties quoted relate only to $\Delta \chi_{\text {critical }}^{2}=1$. However, this region is strongly determined by the underlying model used in the tune.

A covariance matrix is also obtained through the inversion of the Hessian of the log-likelihood function at the best-fit parameter point. The corresponding correlation matrices are presented in Tables IX(a) and IX(b) for the tunes of all four different cross-section model constructions used in this work (see the correlation matrices in Figs. 15 and 16 for a graphical interpretation). An example of the propagation of model uncertainties from the Professor output to the GENIE comparisons framework is shown in Fig. 17.

Joint $\Delta \chi_{\text {profile }}^{2}\left(\theta_{i}, \theta_{j}\right)$ functions, constructed by fixing two parameters at a grid of values and minimizing and $\Delta \chi^{2}(\boldsymbol{\theta}, \boldsymbol{f})$ with respect to all other new parameters, are shown in Fig. 18 for selected sets of parameters. In Fig. 18, we can see that the coverage of the parameter space for the $68 \%$ and $95 \%$ confidence level lines is wider for the G18_01a(/b) tunes. This characteristic is again not related with how well we can constrain the parameters from the data, but with the capability of the models to accommodate this data in each model implementation.

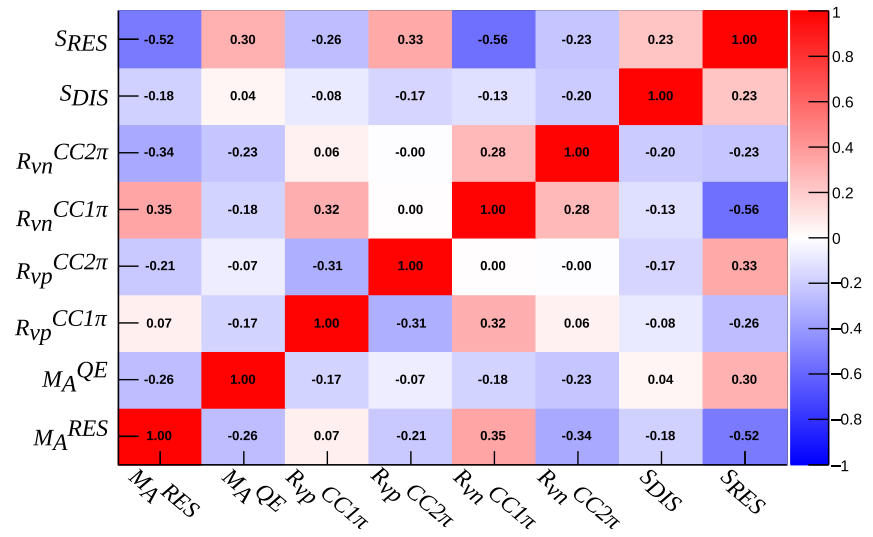

FIG. 15. Parameter correlation matrix from the GENIE fit using the G18_01a(/b) CMC correlation matrix.

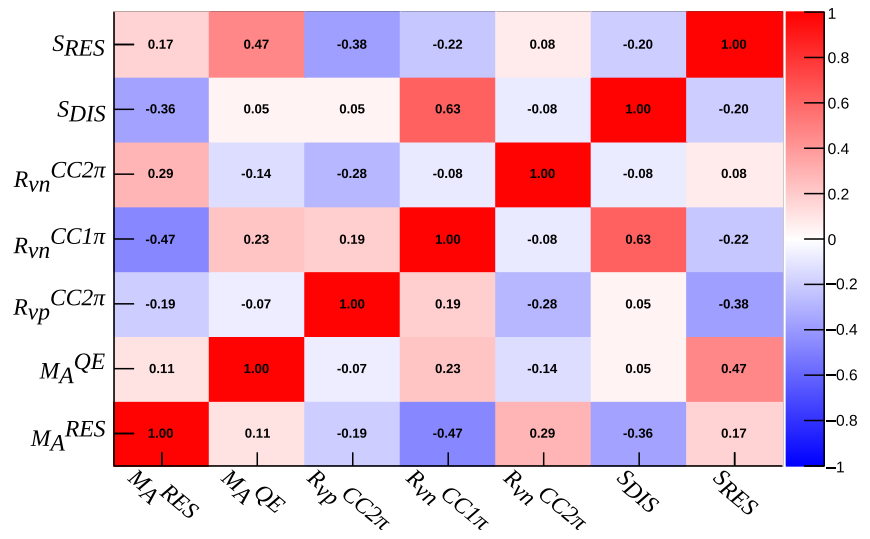

FIG. 16. Parameter correlation matrix from the GENIE fit using the G18_02a(/b) CMC correlation matrix. 


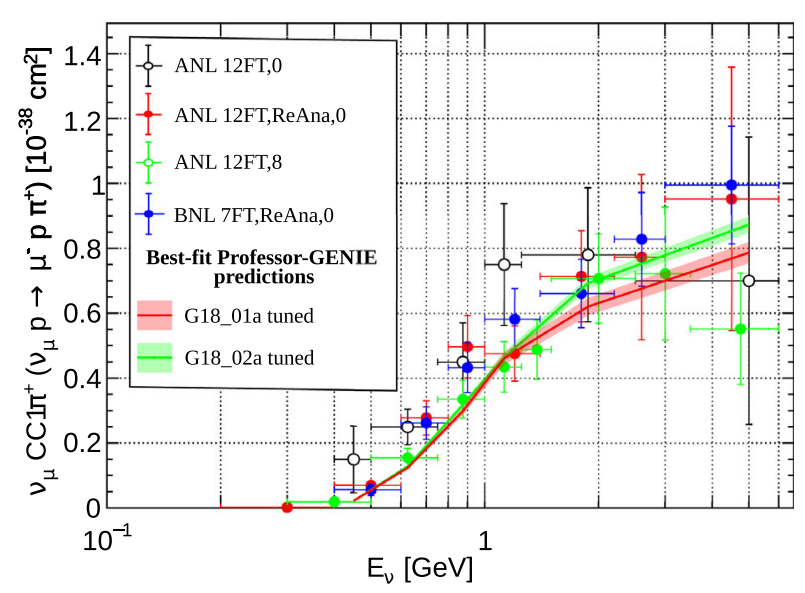

(a) $\nu_{\mu} \mathrm{CC} 1 \pi^{+}$comparison.

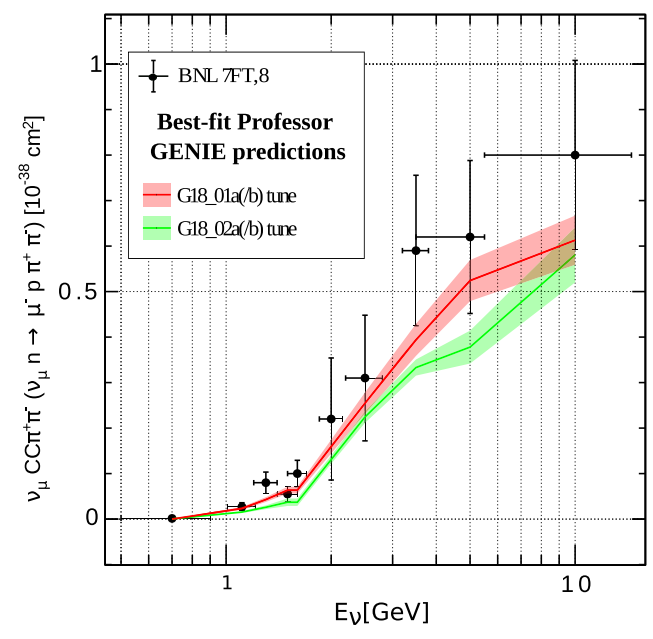

(b) $\nu_{\mu} \mathrm{CC} \pi^{+} \pi^{-}$comparison.

FIG. 17. Comparisons of neutrino data against predictions obtained from the Professor-GENIE parametrization at the best-fit value. The uncertainties of the tune are propagated to the prediction considering the full covariance matrix.

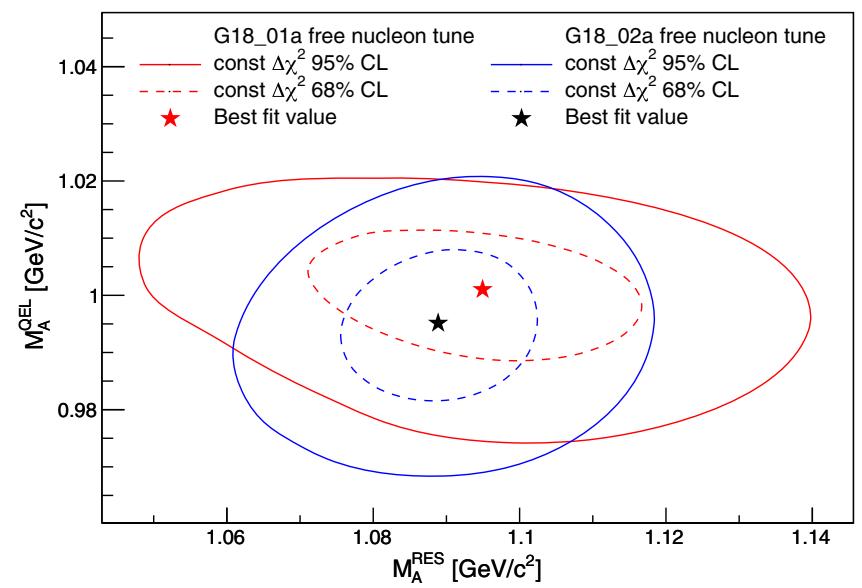

(a) Contour $M_{A}^{\mathrm{RES}}$ vs $M_{A}^{\mathrm{QE}}$

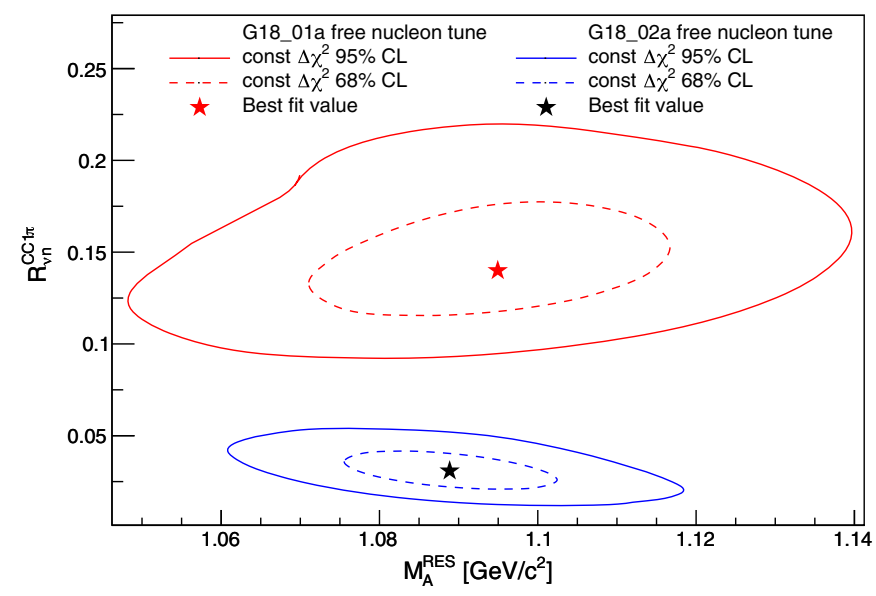

(c) Contour $M_{A}^{\mathrm{RES}}$ vs $R_{\nu n}^{\mathrm{CC} 1 \pi}$

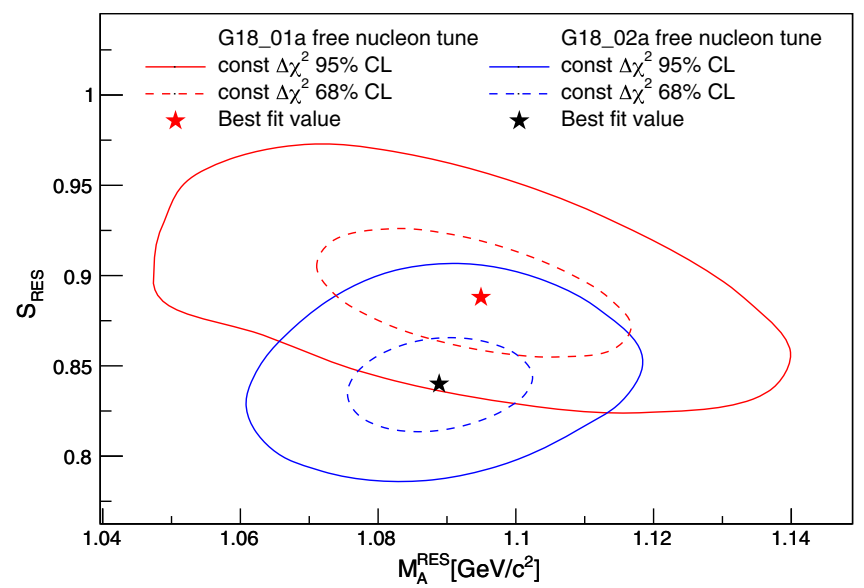

(b) Contour $M_{A}^{\mathrm{RES}}$ vs $S_{\mathrm{RES}}$

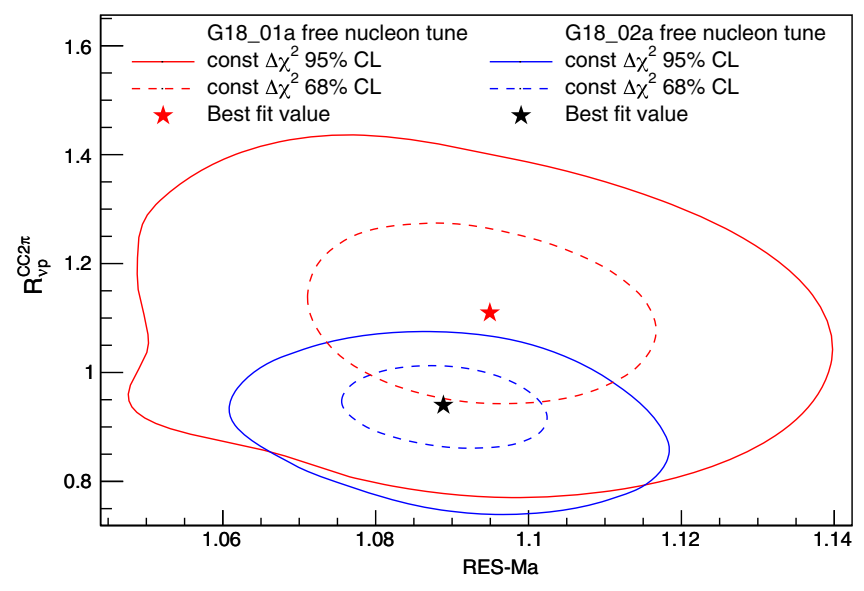

(d) Contour $M_{A}^{\mathrm{RES}}$ vs $R_{\nu p}^{\mathrm{CC} 2 \pi}$

FIG. 18. Joint $\Delta \chi_{\text {profile }}^{2}\left(\theta_{i}, \theta_{j}\right)$ functions obtained by fixing the two parameters under study and minimizing $\Delta \chi^{2}(\vec{\theta}, \vec{f})$ with respect to the other parameters in the tune. The contours for both tunes are shown for each case as well as the best-fit values of each tune. 


\section{CONCLUSIONS}

GENIE has released a number of comprehensive model configurations (CMCs) which consist of different modeling aspects combined altogether. In previous GENIE versions, there was a preferred default comprehensive model which failed to describe both inclusive and exclusive channels due to unresolved tensions between the data. These tensions, which are crucial to understand for the new generation of neutrino experiments, motivated a careful investigation and retune of the bare-nucleon cross-section model for all GENIE comprehensive models available in GENIE v3. Best-fit values and correlations for several parameters influencing the GENIE bare-nucleon cross sections are released in this paper.

In GENIE v3, we focus on improving understanding of the SIS region by tuning the GENIE CMC predictions on hydrogen and deuterium data from the ANL 12FT, BNL 7FT, BEBC, and FNAL 15FT bubble chamber experiments. The tuning of the nonresonant background takes a central stage in this work in order to remove double counting issues. The SIS region has been tuned against $\nu_{\mu}$ and $\bar{\nu}_{\mu} \mathrm{CC}$ inclusive, quasielastic, one-pion and two-pion integrated cross sections as a function of $E_{\nu}$. Quasielastic data has been introduced to the fit to better constrain the flux of each experiment.

The global fit describes both inclusive and exclusive cross sections simultaneously. Tensions were identified between datasets and interpreted as a need for a better tuning procedure, since difficulties in trying to extract information from these datasets were highlighted in previous attempts [4] [Fig. 8]. The tensions motivated the systematic treatment of correlations between datasets and the inclusion of priors as discussed in the paper.

After the global tune, the inclusive cross section at the $1 \mathrm{GeV}-10 \mathrm{GeV}$ energy region decreased with respect to the historical default predictions. The tuned GENIE predictions of one-pion production cross sections on free nucleons $\left(\nu_{\mu} \mathrm{CC} \mathrm{p} \pi^{+}, \mathrm{n} \pi^{+}, \mathrm{p} \pi^{0}\right.$, and $\left.\mathrm{p} \pi^{+} \pi^{-}\right)$show a decrease in the nonresonant background contribution, improving the agreement with the data. The prediction for two-pion production mechanisms is also in better agreement with data for the $\nu_{\mu} n \rightarrow \mu^{-} p \pi^{+} \pi^{-}$channel by increasing the two-pion production nonresonant background contribution.

This is only the first GENIE paper about a systematic tuning procedure. In the future the Collaboration plans to release tunes against hadronization and nuclear data. Both will address issues that near-future neutrino oscillation experiments will have to face.

\section{ACKNOWLEDGMENTS}

We would like to thank Andy Buckley (University of Glasgow, United Kingdom) and Holger Schultz (Institute of Particle Physics Phenomenology, University of Durham, United Kingdom) for their support interfacing the Professor tool with the software products that underpin the GENIE global analysis. We are also grateful with Luis AlvarezRuso for insightful discussions and the review of this paper. We would like to thank the CC-IN2P3 Computing Center, as well as the Particle Physics Department at Rutherford Appleton Laboratory for providing computing resources and for their support. This work, as well as the ongoing development of several other GENIE physics tunes was enabled through a $\mathrm{PhD}$ studentship funded by STFC through LIV.DAT, the Liverpool Big Data Science Centre for Doctoral Training (Project Reference No. 2021488). The initial conceptual and prototyping work for the development of the GENIE / Professor interfaces, as well as for the development of the GENIE global analysis framework that, currently, underpins several analyses, was supported in part through an Associateship Award by the Institute of Particle Physics Phenomenology, University of Durham. This document was prepared by the GENIE collaboration using the resources of the Fermi National Accelerator Laboratory (Fermilab), a U.S. Department of Energy, Office of Science, HEP User Facility. Fermilab is managed by Fermi Research Alliance, LLC (FRA), acting under Contract No. DE-AC02-07CH11359. Russian Science Foundation Grant No. 18-12-00271.

\section{APPENDIX A: COMPREHENSIVE MODEL CONFIGURATION NAMING CONVENTION}

A comprehensive model configuration is identified by at least a seven-character string in the form,

\section{Gdd_MMv}

where

(a) G is a capital letter string of arbitrary length that identifies the authors of the tune (GENIE).

(b) dd is a number describing the year during which the model configuration was first developed.

(c) $\mathrm{MM}$ is a number $(00,01,02, \ldots)$ identifying a family of model configurations.

(d) $\mathrm{v}$ is a character $(\mathrm{a}, \mathrm{b}, \mathrm{c}, \ldots)$ enumerating different members of the given family of model configurations.

Once a comprehensive model configuration is defined, a number of different tunes may be produced. These may be produced, for example, by (a) incorporating different combinations of experimental data, (b) considering variations in different combinations of our modeling elements (e.g., bare-nucleon cross sections, nuclear-model and nuclear cross sections, neutrino-induced hadronization etc.), (c) considering different degrees of freedom (different parametrizations) for the variation of each model, or (d) incorporating different parameter priors and/or different strategies for eliminating nuisance parameters. A tune is identified by the model configuration name, and additional information enumerating the parameters and datasets. This is at least 14-character string in the form 


\section{Gdd_MMv_PP_xxx,}

where

(a) Gdd_MMv describes the model configuration (see above).

(b) PP is a number identifying the set of tuned parameters. This parameter set is defined uniquely only in the context of a particular model configuration.

(c) $\mathrm{xxx}$ is a number that identifies the dataset used for the model configuration tuning. This may include a unique set of weights associated with each component dataset.

\section{APPENDIX B: TUNES FOR $z$-EXPANSION CMCs}

There is a group of CMCs that are derived from from the G18_10[a-d] ones, by replacing the dipole-axial form factor, used in the calculations of quasielastic cross sections, with the better-motivated $z$-expansion model [89], providing a richer set of degrees of freedom for parametrizing quasielastic model uncertainties. They are labeled G18_10[i-1]. As in all previous families of models, four comprehensive model variations (i)-(l) are constructed by using alternative intranuclear hadron transport models (i) INTRANUKE/hA, (j) INTRANUKE/hN, (k) GEANT4/ Bertini, and (l) INCL++.

A specific tune has not been performed by the GENIE collaboration for these CMCs, yet their tuned versions are available in the generator. The released tunes (G18_10[i-1]_02_11b) are constructed by applying all the values obtained in the fit, Table VII (G18_02a column), with the exception of $M_{A}^{\mathrm{QE}}$. Instead, the $z$-expansion parameters provided in [89] are used for the QEL axial form factor. This is considered good enough for the time being, since the fit targets specifically the SIS region and the uncertainty of the QEL axial form factor is only used to improve the fitting procedure. In addition, the $M_{A}^{\mathrm{QE}}$ fit result is not far from its initial value.

In the future we expect to be able to release a dedicated tune for these CMCs that uses the full z-expansion uncertainty from [89] as a prior in a similar exercise as described in this paper.
[1] C. Andreopoulos et al., The GENIE neutrino Monte Carlo generator, Nucl. Instrum. Methods Phys. Res., Sect. A 614, 87 (2010).

[2] A. Buckley, H. Hoeth, H. Lacker, H. Schulz, and J. E. von Seggern, Systematic event generator tuning for the LHC, Eur. Phys. J. C 65, 331 (2010).

[3] H. Schulz et al., Professor web page, https://professor .hepforge.org (2020).

[4] P. Rodrigues, C. Wilkinson, and K. McFarland, Constraining the GENIE model of neutrino-induced single pion production using reanalyzed bubble chamber data, Eur. Phys. J. C 76, 474 (2016).

[5] D. Rein and L. M. Sehgal, Neutrino-excitation of baryon resonances and single pion production, Ann. Phys. (N.Y.) 133, 79 (1981).

[6] K. S. Kuzmin, V. V. Lyubushkin, and V. A. Naumov, Lepton polarization in neutrino-nucleon interactions, Mod. Phys. Lett. A 19, 2815 (2004); Phys. Part. Nucl. 35, S133 (2004).

[7] K. S. Kuzmin, V. V. Lyubushkin, and V. A. Naumov, Extended Rein-Sehgal model for tau lepton production, Nucl. Phys. B, Proc. Suppl. 139, 158 (2005).

[8] C. Berger and L. M. Sehgal, Lepton mass effects in single pion production by neutrinos, Phys. Rev. D 76, 113004 (2007); Erratum, Phys. Rev. D 77, 059901 (2008).

[9] A. Bodek and U. Yang, Modeling deep inelastic cross sections in the few GeV region, Nucl. Phys. B, Proc. Suppl. 112, 70 (2002).

[10] T. Yang, C. Andreopoulos, H. Gallagher, K. Hoffmann, and P. Kehayias, A hadronization model for few-GeV neutrino interactions, Eur. Phys. J. C 63, 1 (2009).
[11] J. Mandula, C. Rebbi, R. Slansky, J. Weyers, and G. Zweig, Duality and the Hadron Spectrum, Phys. Rev. Lett. 22, 1147 (1969).

[12] H. Grässler et al., Multiplicities of secondary hadrons produced in $\nu p$ and $\bar{\nu}$ charged current interactions, Nucl. Phys. B223, 269 (1983).

[13] D. Zieminska et al., Charged particle multiplicity distributions in $\nu n$ and $\nu p$ charged current interactions, Phys. Rev. D 27, 47 (1983).

[14] S. Barlag et al. (Amsterdam-Bologna-Padova-PisaSaclay-Torino Collaboration), Charged hadron multiplicities in high energy $\bar{\nu}_{\mu} n$ and $\bar{\nu}_{\mu} p$ interactions, Z. Phys. C 11, 283 (1982); Erratum, Z. Phys. C 14, 281 (1982).

[15] A. Bodek and J. L. Ritchie, Fermi-motion effects in deepinelastic lepton scattering from nuclear targets, Phys. Rev. D 23, 1070 (1981).

[16] J. Nieves, I. R. Simo, and M. J. V. Vacas, Inclusive chargedcurrent neutrino-nucleus reactions, Phys. Rev. C 83, 045501 (2011).

[17] C. Llewellyn Smith, Neutrino reactions at accelerator energies, Phys. Rep. 3, 261 (1972).

[18] D. Rein and L. M. Sehgal, Coherent $\pi^{0}$ production in neutrino reactions, Nucl. Phys. B223, 29 (1983).

[19] L. A. Ahrens et al., Measurement of neutrino-proton and antineutrino-proton elastic scattering, Phys. Rev. D 35, 785 (1987).

[20] S. Kovalenko, Quasielastic neutrino production of charmed baryons from the point of view of local duality, Sov. J. Nucl. Phys. 52, 934 (1990), https://inspirehep.net/literature/ 299416. 
[21] M. A. G. Aivazis, W.-K. Tung, and F. I. Olness, Next-toleading order QCD formulation of deep inelastic scattering, in Proceedings, The Vancouver Meeting, Particles \& Fields'91: Vancouver, Canada, August 18-22, 1991 (1991), pp. 663-665.

[22] D. Rein, Diffractive pion production in neutrino reactions, Nucl. Phys. B278, 61 (1986).

[23] A. Pais, Weak interactions at high energies, Ann. Phys. (N.Y.) 63, 361 (1971).

[24] M. Rafi Alam, I. Ruiz Simo, M. Sajjad Athar, and M. J. Vicente Vacas, Weak kaon production off the nucleon, Phys. Rev. D 82, 033001 (2010).

[25] S. Dytman, Y. Hayato, R. Raboanary, J. T. Sobczyk, J. TenaVidal, and N. Vololoniaina, Comparison of validation methods of simulations for final state interactions in hadron production experiments, arXiv:2103.07535.

[26] S. Agostinelli et al., Geant4a simulation toolkit, Nucl. Instrum. Methods Phys. Res., Sect. A 506, 250 (2003).

[27] A. Heikkinen, N. Stepanov, and J. P. Wellisch, Bertini intranuclear cascade implementation in GEANT4, eConf C0303241, MOMT008 (2003), http://cds.cern.ch/record/ 619377.

[28] D. Mancusi, A. Boudard, J. Carbonell, J. Cugnon, J.-C. David, and S. Leray, Improving the description of protoninduced one-nucleon removal in intranuclear-cascade models, Phys. Rev. C 91, 034602 (2015).

[29] J. Schwehr, D. Cherdack, and R. Gran, Genie implementation of ific valencia model for qe-like $2 \mathrm{p} 2 \mathrm{~h}$ neutrino-nucleus cross section, arXiv:1601.02038.

[30] S. J. Barish, J. Campbell, G. Charlton, Y. Cho, M. Derrick, R. Engelmann, L. G. Hyman, D. Jankowski, A. Mann, B. Musgrave, P. Schreiner, P. F. Schultz, R. Singer, M. Szczekowski, T. Wangler, H. Yuta, V. E. Barnes, D. D. Carmony, A. F. Garfinkel, and G. M. Radecky, Study of neutrino interactions in hydrogen and deuterium: Description of the experiment and study of the reaction $\nu+d \rightarrow \mu^{-}+p+p_{s}$, Phys. Rev. D 16, 3103 (1977).

[31] P. C. Bosetti et al. (Aachen-Bonn-CERN-London-OxfordSaclay Collaboration), Total cross sections for chargedcurrent neutrino and anti-neutrino interactions in BEBC in the energy range 20-200 GeV, Phys. Lett. 70B, 273 (1977).

[32] P. Bosetti et al. (Aachen-Bonn-CERN-Democritos-LondonOxford-Saclay Collaboration), Total cross-sections for $\nu_{\mu}$ and $\bar{\nu}_{\mu}$ charged-current interactions between 20 and $200 \mathrm{GeV}$, Phys. Lett. 110B, 167 (1982).

[33] C. Baltay et al., Cross-Sectons and Scaling Variable Distributions of Neutral and Charged Current Neutrino Nucleon Interactions from a Low-Energy Narrow Band Beam, Phys. Rev. Lett. 44, 916 (1980).

[34] W. G. Seligman, A next-to-leading order QCD analysis of neutrino-iron structure functions at the Tevatron, Ph.D. thesis, Nevis Labs, Columbia University, 1997.

[35] M. Jonker et al. (CHARM Collaboration), Experimental study of neutral-current and charged-current neutrino cross sections, Phys. Lett. 99B, 265 (1981); Erratum, Phys. Lett. 100, 520 (1981), http://cds.cern.ch/record/134078.

[36] T. Kitagaki et al., Charged-current exclusive pion production in neutrino-deuterium interactions, Phys. Rev. D 34, 2554 (1986).
[37] T. Eichten et al., Measurement of the neutrino-nucleon antineutrino-nucleon total cross sections, Phys. Lett. 46B, 274 (1973).

[38] J. G. Morfin et al. (Gargamelle SPS Experiment Collaboration), Total cross-sections and nucleon structure functions in the Gargamelle SPS neutrino/antineutrino experiment, Phys. Lett. 104B, 235 (1981).

[39] A. S. Vovenko, Total cross section measurements for $\nu_{\mu}, \bar{\nu}_{\mu}$ interactions in 3-30 GeV energy range with IHEP-JINR detector and future plans, Nucl. Phys. B, Proc. Suppl. 112, 116 (2002).

[40] V. Lyubushkin et al. (NOMAD Collaboration), A study of quasi-elastic muon neutrino and antineutrino scattering in the NOMAD experiment, Eur. Phys. J. C 63, 355 (2009).

[41] P. Adamson et al. (MINOS Collaboration), Neutrino and antineutrino inclusive charged-current cross section measurements with the MINOS near detector, Phys. Rev. D 81, 072002 (2010).

[42] S. J. Barish et al., Study of neutrino interactions in hydrogen and deuterium: Inelastic charged current reactions, Phys. Rev. D 19, 2521 (1979).

[43] D. C. Colley et al., Cross-sections for charged current $\nu$ and $\bar{\nu}$ interactions in the energy range 10 to $50 \mathrm{GeV}, \mathrm{Z}$. Phys. C 2, 187 (1979).

[44] M. A. Parker et al. (BEBC TST Neutrino Collaboration), A comparison of charged current cross sections and structure functions for neutrino and anti-neutrinos beams on Hydrogen and Neon, Nucl. Phys. B232, 1 (1984).

[45] N. J. Baker, P. L. Connolly, S. A. Kahn, M. J. Murtagh, R. B. Palmer, N. P. Samios, and M. Tanaka, Total cross sections for $\nu_{\mu} n$ and $\nu_{\mu} p$ charged-current interactions in the 7-foot bubble chamber, Phys. Rev. D 25, 617 (1982).

[46] D. MacFarlane et al., Nucleon structure functions from high-energy neutrino interactions with iron and QCD results, Z. Phys. C 26, 1 (1984).

[47] J. V. Allaby et al. (CHARM Collaboration), Total cross sections of charged-current neutrino and antineutrino interactions on isoscalar nuclei, Z. Phys. C 38, 403 (1988).

[48] N. J. Baker et al., Measurement of the $\nu_{\mu}$ Charged-Current Cross Section, Phys. Rev. Lett. 51, 735 (1983).

[49] S. Ciampolillo et al. (Gargamelle Neutrino Propane Experiment Collaboration), Total cross-section for neutrino charged current interactions at 3 and $9 \mathrm{GeV}$, Phys. Lett. 84B, 281 (1979).

[50] A. E. Asratian et al., Total antineutrino-nucleon charged current cross section in the energy range 10-50 GeV, Phys. Lett. 137B, 122 (1984).

[51] V. B. Anikeev et al., Total cross-section measurements for $\nu_{\mu}, \bar{\nu}_{\mu}$ interactions in 3-30 GeV energy range with IHEPJINR neutrino detector, Z. Phys. C 70, 39 (1996).

[52] D. S. Baranov et al., Measurements of the $\nu_{\mu} N$ total cross section at 2-30 GeV in SKAT neutrino experiment, Phys. Lett. 81B, 255 (1979).

[53] Y. Nakajima et al. (SciBooNE Collaboration), Measurement of inclusive charged current interactions on carbon in a fewGeV neutrino beam, Phys. Rev. D 83, 012005 (2011).

[54] G. Fanourakis, L. K. Resvanis, G. Grammatikakis, P. Tsilimigras, A. Vayaki, U. Camerini, W. F. Fry, R. J. Loveless, J.H. Mapp, and D. D. Reeder, Study of 
low-energy antineutrino interactions on protons, Phys. Rev. D 21, 562 (1980).

[55] G. N. Taylor, R. J. Cence, F. A. Harris, M. D. Jones, S. I. Parker, M. W. Peters, V.Z. Peterson, V. J. Stenger, H. C. Ballagh, H. H. Bingham, T. Lawry, J. Lys, M. L. Stevenson, G. P. Yost, D. Gee, F. R. Huson, E. Schmidt, W. Smart, and E. Treadwell, $\bar{\nu}_{\mu^{-}}$Nucleon Charged-Current Total Cross Section for 5-250 GeV, Phys. Rev. Lett. 51, 739 (1983).

[56] O. Erriquez et al., Antineutrino-nucleon total cross section and ratio of antineutrino cross section on neutrons and protons, Phys. Lett. 80B, 309 (1979).

[57] A. E. Asratian et al., Charged current neutrino interactions below $30 \mathrm{GeV}$, Phys. Lett. 76B, 239 (1978).

[58] W. A. Mann et al., Study of the Reaction $\nu+n \rightarrow \mu^{-}+p$, Phys. Rev. Lett. 31, 844 (1973).

[59] D. Allasia et al., Investigation of exclusive channels in $\nu / \bar{\nu}$-deuteron charged current interactions, Nucl. Phys. B343, 285 (1990).

[60] T. Kitagaki et al., High-energy quasielastic $\nu_{\mu} n \rightarrow \mu^{-} p$ scattering in deuterium, Phys. Rev. D 28, 436 (1983).

[61] S. V. Belikov et al., Quasielastic $\nu_{\mu} n$ scattering at 3-30 GeV energy, Yad. Fiz. 35, 59 (1982).

[62] J. Brunner et al. (SKAT Collaboration), Quasielastic nucleon and hyperon production by neutrons and antineutrinos with energies below $30 \mathrm{GeV}$, Z. Phys. C 45, 551 (1990).

[63] N. J. Baker, A. M. Cnops, P. L. Connolly, S. A. Kahn, H. G. Kirk, M. J. Murtagh, R. B. Palmer, N. P. Samios, and M. Tanaka, Quasielastic neutrino scattering: A measurement of the weak nucleon axial-vector form factor, Phys. Rev. D 23, 2499 (1981).

[64] S. Bonetti, G. Carnesecchi, D. Cavalli, P. Negri, A. Pullia, M. Rollier, F. Romano, and R. Schira, Study of Quasi-elastic Reactions of $\nu$ and $\bar{\nu}$ in Gargamelle, Nuovo Cimento A 38, 260 (1977).

[65] S. V. Belikov et al., Restraints on parameters of oscillations of muon neutrinos from quasielastic scattering data., Yad. Fiz. 41, 919 (1985), https://inspirehep.net/literature/219091.

[66] N. Armenise et al., Charged current elastic antineutrino interactions in propane, Nucl. Phys. B152, 365 (1979).

[67] J. Campbell et al., Study of the Reaction $\nu p \rightarrow \mu^{-} \pi^{+} p$, Phys. Rev. Lett. 30, 335 (1973).

[68] G. M. Radecky et al., Study of single-pion production by weak charged currents in low-energy $\nu d$ interactions, Phys. Rev. D 25, 1161 (1982); Erratum, Phys. Rev. D 26, 3297 (1982).

[69] C. Wilkinson, P. Rodrigues, S. Cartwright, L. Thompson, and K. McFarland, Reanalysis of bubble chamber measurements of muon-neutrino induced single pion production, Phys. Rev. D 90, 112017 (2014).

[70] W. Lerche et al. (Gargamelle Neutrino Propane Experiment Collaboration), Experimental study of the reaction $\nu p \rightarrow \mu^{-} \pi^{+} p$, Phys. Lett. 78B, 510 (1978).

[71] P. Allen et al. (Aachen-Bonn-CERN-Munich-Oxford Collaboration), Single $\pi^{+}$production in charged current neutrino-hydrogen interactions, Nucl. Phys. B176, 269 (1980).
[72] J. Bell et al., Study of the Reactions $\nu p \rightarrow \mu^{-} \Delta^{++}$at High Energies and Comparisons with Theory, Phys. Rev. Lett. 41, 1012 (1978).

[73] P. Allen et al. (Aachen-Birmingham-Bonn-CERN-LondonMunich-Oxford), A study of single meson production in neutrino and antineutrinos charged-current interactions on protons, Nucl. Phys. B264, 221 (1986).

[74] S. J. Barish et al., Study of the reaction $\bar{\nu}_{\mu} p \rightarrow \mu^{+} p \pi^{-}$, Phys. Lett. 91B, 161 (1980).

[75] T. Kitagaki et al., Neutrino Flux and Total Charged-Current Cross Sections in High-Energy Neutrino-Deuterium Interactions, Phys. Rev. Lett. 49, 98 (1982).

[76] D. Day et al., Radecky, Study of $\nu d$ charged-current twopion production in the threshold region, Phys. Rev. D 28, 2714 (1983).

[77] J. P. Berge et al., Inclusive negative-hadron production from high-energy $\bar{\nu}$-nucleus charged-current interactions, Phys. Rev. D 18, 3905 (1978).

[78] J. Bell et al., Cross-Section Measurements for the Reactions $\nu p \rightarrow \mu^{-} \pi^{+} p$ and $\nu p \rightarrow \mu^{-} K^{+} p$ at High Energies, Phys. Rev. Lett. 41, 1008 (1978).

[79] P. Zyla and others (Particle Data Group Collaboration), Review of particle physics, Prog. Theor. Exp. Phys. 2020, 083C01 (2020).

[80] K. S. Kuzmin, V. V. Lyubushkin, and V. A. Naumov, Axial masses in quasielastic neutrino scattering and single-pion neutrinoproduction on nucleons and nuclei, Acta Phys. Pol. B 37, 2337 (2006).

[81] K. S. Kuzmin, V. V. Lyubushkin, and V. A. Naumov, Quasielastic axial-vector mass from experiments on neutrinonucleus scattering, Eur. Phys. J. C 54, 517 (2008), https:// inspirehep.net/literature/719586.

[82] A. Bodek, S. Avvakumov, R. Bradford, and H. S. Budd, Vector and axial nucleon form factors: A duality constrained parameterization, Eur. Phys. J. C 53, 349 (2008).

[83] $\boldsymbol{\theta}=\left(W_{\text {cut }}, M_{A}^{\mathrm{QE}}, M_{A}^{\mathrm{RES}}, R_{\nu p}^{\mathrm{CC} 1 \pi}, R_{\nu p}^{\mathrm{CC} 2 \pi}, R_{\nu n}^{\mathrm{CC} 1 \pi}, R_{\nu n}^{\mathrm{CC} 2 \pi}, S_{\mathrm{RES}}\right.$, $S_{\text {DIS }}$.

[84] For instance, BEBC data on $\nu_{\mu}$ will have an associated nuisance parameter, which will be different from the one applied to $\mathrm{BEBC} \bar{\nu}_{\mu}$ data.

[85] The best agreement with all high energy data requires $S_{\text {DIS }} \sim 1$.

[86] A. Buckley, H. Hoeth, H. Lacker, H. Schulz, and J. E. von Seggern, Systematic event generator tuning for the LHC, Eur. Phys. J. C 65, 331 (2010).

[87] Note that in the default GENIE tune, there was no method to include correlation between datasets coming from the same experiment.

[88] M. Betancourt et al., Comparisons and challenges of modern neutrino scattering experiments (tensions2016 report), Phys. Rep. 773-774, 1 (2018), comparisons and challenges of modern neutrino scattering experiments (TENSIONS2016 report).

[89] A. S. Meyer, M. Betancourt, R. Gran, and R. J. Hill, Deuterium target data for precision neutrino-nucleus cross sections, Phys. Rev. D 93, 113015 (2016). 